\title{
Force Fields for Macromolecular Assemblies Containing Diketopyrrolopyrrole and Thiophene
}

Jiang, L., Rogers, D.M., Hirst, J.D., Do, H.

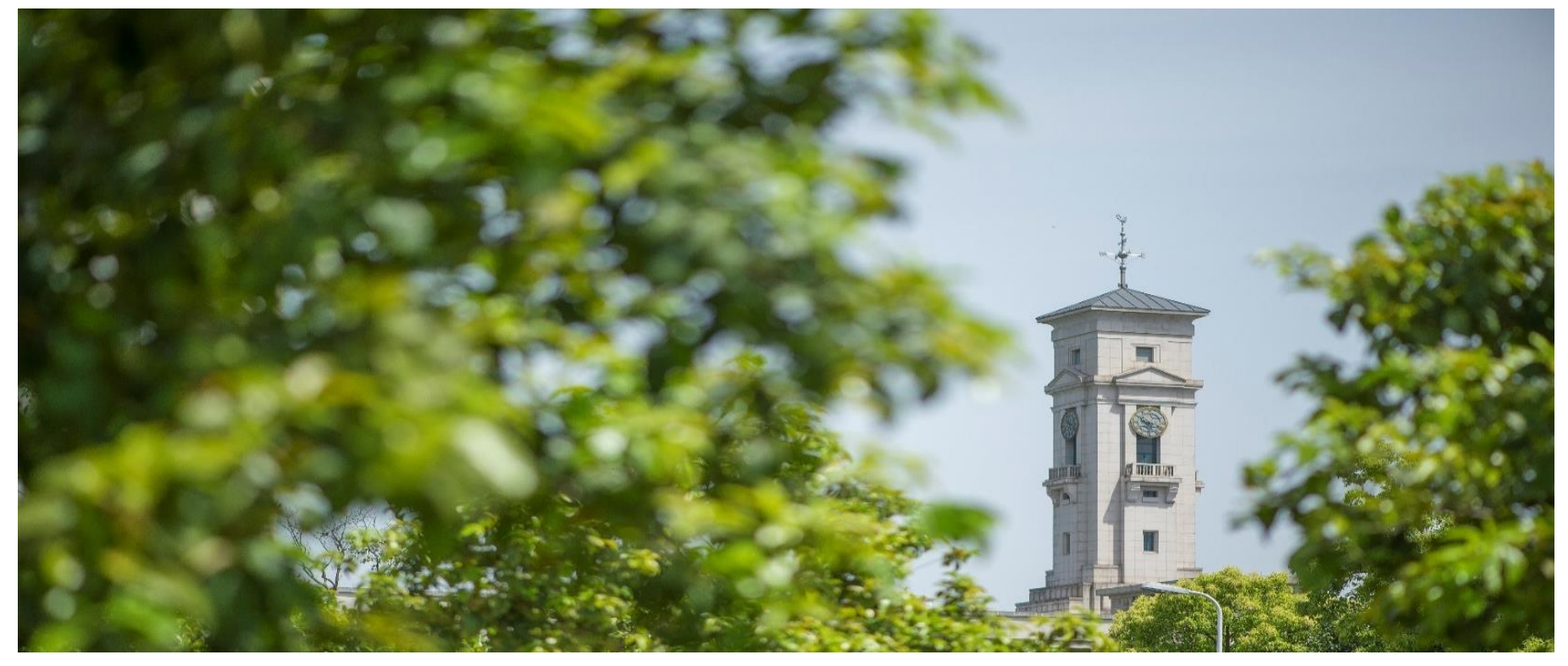


University of Nottingham Ningbo China, 199 Taikang East Road, Ningbo, 315100, Zhejiang, China.

First published 2020

This work is made available under the terms of the Creative Commons Attribution 4.0 International License:

http://creativecommons.org/licenses/by/4.0

The work is licenced to the University of Nottingham Ningbo China under the Global University Publication Licence:

https://www.nottingham.edu.cn/en/library/documents/researchsupport/global-university-publications-licence-2.0.pdf 


\title{
Force Fields for Macromolecular Assemblies Containing Diketopyrrolopyrrole and Thiophene
}

\author{
Ling Jiang, ${ }^{\dagger}$ David M. Rogers, ${ }^{\ddagger}$ Jonathan D. Hirst, ${ }^{\ddagger}$ and Hainam Do*,+, \\ $\dagger$ Department of Chemical and Environmental Engineering, University of Nottingham \\ Ningbo China, Ningbo 315100, China \\ $\ddagger$ School of Chemistry, University of Nottingham, University Park, Nottingham, NG7 2RD, \\ $U K$ \\ 9New Materials Institute, University of Nottingham Ningbo China, Ningbo 315042, China \\ E-mail: hainam.do@nottingham.edu.cn
}

\begin{abstract}
Utilising a force matching procedure, we parameterise new force fields systematically for large conjugated systems. We model both conjugated polymers and molecular crystals that contain diketopyrrolopyrrole, thiophene, and thieno[3,2-b]thiophene units. These systems have recently been found to have low band gaps, which exhibit high efficiency for photovoltaic devices. The equilibrium structures, forces and energies of the building block chromophores: diketopyrrolopyrrole thiophene, and thieno[3,2b]thiophene computed using our parameters are comparable to those computed using the reference electronic structure method. We assess the suitability of this new force field for electronic property calculations by comparing the electronic excitation properties computed along classical and ab initio molecular dynamics trajectories. For both trajectories, we find similar distributions of TDDFT calculated excitation energies and
\end{abstract}


oscillator strengths for the building block chromophore diketopyrrolopyrrole-thieno[3,2b]thiophene. The structural, dynamical, and electronic properties of the macromolecular assemblies built upon these chromophores are characterised. For both polymers and molecular crystals, pronounced peaks around $0^{\circ}$ or $180^{\circ}$ are observed for the torsions between chromophores under ambient conditions. The high planarity in these systems can promote local ordering and $\pi-\pi$ stacking, thereby potentially facilitating charge transport across these materials. For the model conducting polymers, we found that the fluctuations in the density of states per chain per monomer is neglegibly small and does not vary significantly with chains comprising 20 to 40 monomers. Analysis of the electron-hole distributions and the transition density matrices indicates that the delocalised length is approximately four to six monomers, which is in good agreement with other theoretical and experimental studies of different conducting polymers. For the molecular crystals, our investigation of the characteristic timescale of the fluctuation in the excitonic couplings shows that low frequency vibration below $10 \mathrm{~cm}^{-1}$ is observed for the nearest neighbors. These observations are in line with previous studies on other molecular crystals, in which low frequency vibrations are believed to be responsible for the large modulation of the excitonic coupling. Thus, our approach and the new force fields provide a direct route for studying the structure-property relations and the molecular level origins of the high-efficiency of these classes of materials.

\section{Introduction}

Semiconductors based on small organic molecules and conjugated polymers offer the opportunity for large-area deposition on substrates and they can be chemically tuned or functionalized for many specific applications. ${ }^{1}$ In the last two decades, they have emerged as excellent materials for displays, solid-state lighting, sensors and solar cells. ${ }^{2-5}$ Their main advantages are the significant reduction in production cost compared to their organic counterparts and their processes can be easily scaled-up, since organic materials can be solution-processed. 
Conjugated organic molecules exhibit a wealth of structures and properties, due to the range of possible molecular constituents. They can be completely disordered polymers formed by interlaced chains, partially ordered polymers, polycrystalline small molecule-based structures, or small molecule-based single crystals. ${ }^{1,6}$ The detailed characterisation of the structure of conjugated organic assemblies remains a challenging task, often requiring simplified and qualitative morphological descriptions. In addition, the variety of these materials, their possible structures, and their complexity hinder the full elucidation of the relationship between the structures of conjugated organic assemblies and their electronic properties. ${ }^{6,7}$

To investigate the fundamental processes that determine the efficiency of charge transport in organic semiconductors, many efforts have been devoted to understand their structureproperty relationships, ${ }^{8-11}$ including the influence of molecular morphologies on optical and charge transfer events. However, the interplay between microstructure and electronic properties in these materials still remains unclear. ${ }^{12,13}$ Amorphous materials or poly-crystalline materials especially are poorly understood. ${ }^{6}$ In particular, a molecular level comprehension of the exciton transport and charge generation mechanisms is crucial, for example, to the rational design and enhancement of conjugated organic semiconductors for highly efficient photovoltaic devices. Theoretical and computational approaches can play a major role in the study of this important aspect, because they provide direct access to the microscopic and electronic properties of materials.

Molecular simulations offer a direct route to explore the relationship between molecular morphology and charge mobility. Amongst several morphology simulation techniques, ${ }^{14}$ classical molecular dynamics (MD) is the most popular approach, as it gives atomic detail comparable with experimental observations. In the last decade MD simulations have penetrated into the study of optical and electronic properties of materials and biomolecules, where they are primarily used to sample the equilibrium structures explored by the system 
of interest. ${ }^{7,15-17}$ These equilibrium trajectories are utilised in large-scale quantum chemistry calculations to correlate the local structure with the observable electronic structure properties. ${ }^{18}$ Consequently, it is vital that the force fields (FFs) used to describe molecular motion of the system are accurate enough to reproduce experiments. The main concern here is the mismatch in the FFs describing the interactions at the classical level and the electronic structure methods employed for subsequent quantum chemistry calculations.

Historically, FFs employed in classical MD simulations have been designed mainly for biochemical systems, e.g. protein simulations. Although these FFs contain parameters that are transferable to conjugated systems, many aspects require a careful parameterisation due to the conjugation. ${ }^{19}$ For large conjugated systems such as polymers, FFs need to employ many atom types and parameters. Generally, the FFs used to describe the motions of molecular systems are seldom parameterised to reproduce electronic processes, such as electronic excitations, which are sensitive to the underlying description of the molecular structure. It still remains a challenge to find satisfactorily accurate FFs for applications where MD is the preliminary step toward the study of electronic structure properties. ${ }^{19-21}$

Most existing procedures ${ }^{22-25}$ are general tools for constructing FFs for small organic molecules, either in the gas phase or in a continuum solvent. When applied to specific problems in condensed phases, these FFs may not be directly transferable to the systems under investigation. For example, several recent studies of excitation energy transfer in light-harvesting (LH) complexes have shown that FFs used to describe the chromophores have a great influence on the resulting spectral density. ${ }^{26-28}$ This problem is often referred to as the geometry mismatch, i.e. the mismatch between the classical and ab initio potential energy surfaces. As such, Prandi et al. proposed a new set of parameters for the different carotenoids found in the LH complexes of photosystem II, optimised to reproduce electronic excitation properties obtained by DFT. ${ }^{29}$ To investigate this further, Andreussi 
et $a l .{ }^{20}$ systematically checked the feasibility of using classical FFs for quantum mechanics applications. They concluded that for photoinduced processes as an example, poor results are obtained from standard transferable FFs. Thus, in order to mitigate the mismatch in the geometry, the classical potential energy surface and/or its gradients of the system of interest should be mapped onto the ab initio ones by optimising the FFs. This would also implicitly incorporate many-body effects, which is crucial in the study of condensed phase systems. To address this, Ercolessi and Adams proposed the force-matching (FM) approach, ${ }^{30}$ which was first employed to develop interatomic potentials that reproduce those computed from first principles. To overcome the computational cost incurred by using ab initio MD or QM/MM, Wang and coworkers introduced the adaptive force matching technique. ${ }^{31,32}$ Instead of using ab initio MD, the adaptive force matching approach uses MM FFs to generate ensembles of equilibrium structures followed by decoupled QM/MM calculations on each configuration with the environment represented by point charges. The procedure starts with a set of guessed parameters and is repeated until convergence is reached. Since then, the method has been applied to systems including transition metal complexes, ${ }^{33,34}$ anions, ${ }^{35}$ ionic liquids, ${ }^{36}$ and microporous materials. ${ }^{37}$ Recently, the force-matching technique has also been employed to develop FFs for water, ${ }^{38,39}$ water on graphene surface, ${ }^{40}$ and explicit three-body interactions for molten carbon. ${ }^{41}$

In this work, we extend the adaptive force matching technique to rapidly parameterize FFs for complex conjugated systems. We develop a strategy for fragmenting the "larger" molecule into smaller segments, obtain their parameters, and then assemble them together to derive the FFs for the "larger" molecule. This was a challenge, which was presented, but not achieved, in the previous work by Do and Troisi. ${ }^{42}$ Do and Troisi ${ }^{42}$ reported parameters for the monomer DPP and for the chromophore-triple DPP-TT-T, but for no other species, precluding the characterisation of the dynamics of the polymer and crystal systems that we study herein. Moreover, the suitability of the force field for electronic property calculations 
by comparing the electronic excitation properties computed along classical and ab initio molecular dynamics trajectories was not assessed. This is important and should be tested, at least, for the monomer. In the current study, we build on and go beyond the earlier work $^{42}$ to address three important aspects. (i) In the current work, we fragment the "larger" molecule into smaller segments, obtain their parameters, and then assemble them together to derive the FF for the"larger" molecule. This was a challenge, which was presented, but not achieved for the chromophore-quadruple (the monomer of the model polymer) in the previous work. ${ }^{42}$ (ii) We validate the FF with the first MD simulation of the model polymer (for a single chain 1-mer) by comparing against counterpart ab initio MD simulations. (iii) We then predict the structural and the electronic excitation properties for the model polymer and also for a newly synthesised molecular crystal. The thrust of our approach is to obtain parameters consistent with any pre-defined electronic structure calculation method. As an example, we parametrize FFs for conducting polymers and molecular crystals containing diketopyrrolopyrrole (DPP), thiophene (T), and thieno[3,2-b]thiophene (TT). The DPP units have strong intermolecular donor-acceptor interactions, which can promote selfassembly of the polymer and enhance the charge transport. ${ }^{43}$ In addition, the incorporation of DPP into an oligothiophene or polythiophene backbone results in low band gap conjugated systems exhibiting high efficiency for photovoltaic devices. ${ }^{44}$ Thus, FFs that generate accurate equilibrium structures via MD simulations of these systems are crucial for analyzing and understanding the structure-property relationships of conjugated semiconductors.

\section{Methods}

The idea behind the force-matching procedure is to find a set of parameters that minimizes the differences between the classical forces and the $a b$ initio forces. Here, the latter can be computed using any reference electronic structure method. Generally, this is chosen to be consistent with the method that is employed in subsequent quantum chemistry calculations. 
In addition, an appropriate $\mathrm{FF}$ that best represents the system of interest has to be determined in advance. The errors between the two types of forces are computed from a set of uncorrelated configurations generated from MD simulations during the production runs. In the approach we use, ${ }^{42}$ a set of parameters $\left\{p_{j}\right\}$ is optimized by minimizing an objective function $O\left(\left\{p_{j}\right\}\right)$, which is defined as the root mean square deviation (RMSD) of two types of forces:

$$
O\left(\left\{p_{j}\right\}\right)=\sqrt{\frac{1}{3 M N} \sum_{k=1}^{M} \sum_{i=1}^{N}\left\|\mathbf{f}_{i, k}^{A B}-\mathbf{f}_{i, k}^{F F}\right\|^{2}}
$$

where $N$ is the number of atoms in the molecule and $M$ is the number of configurations

used. $\mathbf{f}_{i, k}^{A B}$ denotes the $a b$ initio forces exerted on atom $i$ of configuration $j$ and $\mathbf{f}_{i, k}^{F F}$ are the equivalent forces calculated using the FFs. $\|. .$.$\| denote the norm. The optimization is$ carried out using a Monte Carlo (MC) method. Further details can be found in the previous work by Do and Troisi. ${ }^{42}$

\section{Computational Details}

To start with, a set of $M$ equilibrium configurations is generated using an initially "guessed" FF. The atom-type is carefully assigned to take into account the complex chemical structure of the system. As such, we tend to have more atom-types than standard FFs. A description of how the different atom types are chosen can be found in the SI. Apart from the point charges and equilibrium bonds and angles, all other "guessed" parameters are taken from OPLS. ${ }^{24,45-47}$ For missing parameters, we use analogy and quantum chemistry calculations to assign the parameters (e.g. equilibrium bonds and angles). Equilibrium structures are obtained from MD simulations under ambient conditions (i.e. $300 \mathrm{~K}$ and 1 bar). All MD simulations are carried out using the NAMD software. ${ }^{48}$ After an energy minimization of 10000 steps, a 200 ps equilibration run is carried out, followed by a 1 ns production run, using a $1 \mathrm{fs}$ time step. Equilibrium structures are saved during the production run at $2 \mathrm{ps}$ in- 
tervals to form a set of 500 snapshots. $M$ uncorrelated equilibrium configurations are drawn randomly from this set. The number of structures, $M$, used in the optimization process is critical and must be determined beforehand. One should not use too few structures, as that would lead to overfitting. On the other hand, with too many structures the computational cost would increase. To work out the "ideal" number of structures, we follow a suggestion from previous work: ${ }^{42} M>\left(50 \times N_{p}\right) /\left(3 \times N_{a}\right)$, where $N_{p}$ is the number of parameters, $N_{a}$ is the number of atoms in the molecule of interest. We found that a value of $M$ between 100 to 150 seems reasonable.

Ab initio MD simulations are carried out using the Atom Centered Density Matrix Propagation (ADMP) molecular dynamics model,${ }^{49-51}$ as implemented in the Gaussian 16 package. ${ }^{52}$ B3LYP $/ 6-31 \mathrm{G}^{*}$ level of theory and a time step of $1 \mathrm{fs}$ are employed throughout, with an initial equilibration run of $10 \mathrm{ps}$, followed by a production run of $50 \mathrm{ps}$, from which 100 uncorrelated configurations (frames) are obtained by sampling every 0.5 ps. These 100 frames are utilised to compute the electronic excitation properties of the building block chromophores. Gaussian $16^{52}$ was also employed to perform TDDFT calculations, at the B3LYP /6-311G(d,p) level, to compute transition energies and oscillator strengths for 20 singlet excited states for each chromophore using frames from the classical and the ab initio MD.

The ab initio forces (reference forces) are computed with DFT using the B3LYP functional and the $6-31 \mathrm{G}^{*}$ basis set, using the Gaussian 16 package. ${ }^{52}$ Depending on the applications and the methods used in the quantum chemistry calculation stage, other combinations of functionals and basis sets and/or higher level of theory can be employed. In our work, we find B3LYP $/ 6-31 \mathrm{G}^{*}$ is sufficient for the systems under investigation. The optimization procedure starts with computing the initial objective function, $O\left(\left\{p_{j}^{i n i t}\right\}\right)$, using equation 1. The algorithm proceeds by randomly selecting a parameter $p_{j}$ from the set and modifies it by adding a random number $\delta p_{j}$ uniformly distributed between $-\delta p_{j}^{\max }$ and $\delta p_{j}^{\max }$. In other 
words, $\delta p_{j}^{\max }$ is the maximum "displacement" of the parameter $\delta p_{j}$. This value is initialized to $0.3 \times p_{j}$ at the beginning of the optimization, and is adjusted in the course of the process to keep the acceptance rate of the MC moves at about 30\%. The optimization is divided into MC "blocks". Each consists of $100 \times N_{p}$ attempts to randomly select and change a parameter $p_{j}$. The idea here is to allow each parameter to be selected roughly 100 times in each MC block. This number can be adjusted and we found 100 times is sufficient. The acceptance rate is calculated at the end of each block and the maximum "displacement" $\delta p_{j}^{\max }$ is adjusted accordingly. The optimization is converged when $\delta O<10^{-10} \mathrm{kcal} \mathrm{mol}^{-1} \AA^{-1}$, where $\delta O$ is the change in the objective function between two consecutive MC steps. Having completed the optimization process, the parameters are then used as the new guess for the next iteration. In general, two or three iterations are sufficient as shown in the results section.

To investigate the nature of the electronic excitation of the polymer, we analyse the electron-hole (e-h) distributions and the transition densities for the lowest three singlet excitations of a single chain (in the absent of the interchain electronic coupling) using the Multiwfn codes. ${ }^{53}$ In general, electronic excitations are represented as transitions involving multiple, weighted molecular orbitals (MO) pairs. When there is no single dominant MO pair transition to represent the electron and hole, the natural transition orbital (NTO) analysis is often utilised. However, for complex molecular systems, there is no guarantee that a dominating NTO pair exists. Thus, to overcome this, several key indices have been proposed to characterise the electronic excited states. Notable are the $\Lambda$-index,${ }^{54} \Delta$ r-index,${ }^{55}$ and other descriptors e.g. e-h overlapping index $\left(\mathrm{S}_{\mathrm{r}}\right.$-index) and charge transfer length index (D-index) etc. proposed by Lu. ${ }^{53}$

The overlap function $\mathrm{S}_{\mathrm{r}}(\mathbf{r})$ between a hole and an electron can be defined as $\mathrm{S}_{\mathrm{r}}(\mathbf{r})=$ $\sqrt{\rho^{\text {hole }}(\mathbf{r}) \rho^{\text {elec }}(\mathbf{r})}$, where $\rho^{\text {hole }}$ and $\rho^{\text {elec }}$ are the charge densities of the hole and electron, respectively. Thus, the $S_{r}$-index is the integration of the $S_{r}(\mathbf{r})$ function over all space, 
which provides the extent of the overlap between hole and electron. The charge transfer (CT) length can be estimated by computing the distance between the centroid of hole and electron. To compute the $\mathrm{X}$ component of the centroid of the electron, for example, we integrate the $\mathbf{x}$-coordinate of the position vector $\mathbf{r}$ weighted by the electron density i.e. $X_{\text {elec }}=\int x \rho^{\text {elec }}(\mathbf{r}) \mathrm{d} \mathbf{r}$. The total magnitude of CT length is referred to as the D-index and can be computed as D-index $=\sqrt{D_{x}^{2}+D_{y}^{2}+D_{z}^{2}}$, where $D_{x}=X_{\text {elec }}-X_{\text {hole }}, D_{y}=Y_{\text {elec }}-Y_{\text {hole }}$, $D_{z}=Z_{\text {elec }}-Z_{\text {hole }}$. The extent of the spatial distribution of the hole and electron can be characterised by the root mean squared deviation. The x-component of the RMSD of the hole

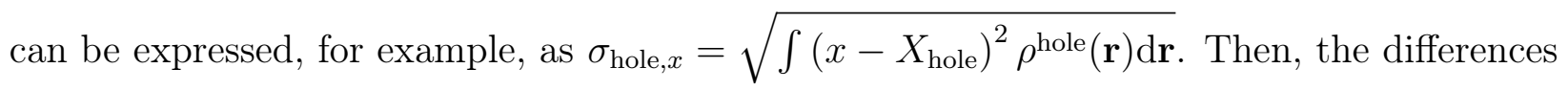
between the RMSD of electron and hole, $\Delta \sigma_{\lambda}$, can be calculated via $\Delta \sigma_{\lambda}=\sigma_{\text {elec, } \lambda}-\sigma_{\text {hole, } \lambda}$, where $\lambda=\{x, y, z\}$. Subsequently, the total difference can be measured via the $\Delta \sigma$-index, which is $\left|\boldsymbol{\sigma}_{\text {elec }}\right|-\left\lceil\boldsymbol{\sigma}_{\text {hole }}\right]$. On the other hand, the H-index, which is given by $\left(\left|\boldsymbol{\sigma}_{\text {elec }}\right|+\right.$ $\left.\left[\boldsymbol{\sigma}_{\text {hole }}\right]\right) / 2$ provides the average degree of spatial extent of the hole and electron distribution in space. For CT excitations, the $t$-index is often employed to describe the separation degree of hole and electron in the CT direction. This descriptor is defined as the difference between the D-index and a quantity called $\mathrm{H}_{\mathrm{CT}}$, which is defined as $H_{C T}=\left|\mathbf{H} \cdot \mathbf{u}_{C T}\right|$. Here, $\mathbf{u}_{C T}$ is a unit vector in the CT direction and can be derived from the centroid of hole and electron, and the component $H_{\lambda}$ of vector $H$ is the average spatial extension of the hole and electron in the $\lambda$-direction, i.e. $H_{\lambda}=\left(\sigma_{\text {elec, } \lambda}+\sigma_{\text {hole }, \lambda}\right) / 2$. In addition, the exciton binding (Coulomb attraction between hole and electron) is another meaningful descriptor that can be utilised to describe the separation of hole and electron.

\section{Force Field Development}

Recently, a series of interesting thienothiophene-flanked diketopyrrolopyrrole (DPPTT)based copolymers with various branched alkyl side-chains have been synthesized. ${ }^{5,56-58}$ How- 
ever, there have not been many reports in the literature regarding reliable FFs to study these polymers. The second system of interest is a molecular crystal also recently synthesized: diethyl 2,2'-(1,4-dioxo-3,6-bis(2-thienyl)pyrrolo[3,4-c]pyrrole-2,5(1H,4H)-diyl)diacetate (CCDC crystal code: UBEQOK). ${ }^{59}$

For all FFs, the general functional expression is the sum of potential energy of the bonded and nonbonded interactions. The bonded part involves terms of bonds, angles and dihedrals, while the nonbonded part comprises van der Waals interactions and electrostatics. The FF parameters needed for the bonded and nonbonded terms are defined for each type of atom. The exact form of these terms may vary depending on the specific FF and system. In this case, the OPLS FF ${ }^{45,60}$ (shown in the Supplementary Information (SI)) is employed. Here, our focus is on the intramolecular components for which the available parameters perform poorly. ${ }^{7,61}$ We employ the corresponding van der Waals parameters from OPLS and compute charges using the RESP method ${ }^{62}$ at the B3LYP/6-31G* level of theory. The intermolecular parameters are kept constant during the optimization.

In this section we shall describe the procedure to construct the FFs for the above mentioned conducting polymers and molecular crystals that contain diketopyrrolopyrrole (DPP), thiophene $(\mathrm{T})$ and thieno[3,2-b]thiophene (TT) chromophores. Instead of optimising the parameters for the whole system in one go as in the work of Do and Troisi, ${ }^{42}$ our strategy is to fragment the "larger" molecule into smaller segments, obtain their parameters, and then assemble them together to derive the FFs for the "larger" molecule. The idea is to parameterize the FFs for each single chromophore (e.g. DPP, TT and T) and utilize them to construct the FFs for larger segments, e.g. segments that contain two or more chromophores. This can be achieved by running the optimization procedure for the "larger" molecules and only allow the missing parameters to be optimized while keeping the rest fixed at those obtained from the single chromophores. In practice, we perform a further optimization for the whole "larger" 
molecules (including sidechains if there is any) to obtain better sets of parameters. These calculations are fairly quick, as the original "guessed" parameters are near optimum. Thus, our fragmentation approach is particularly suitable for large systems with many parameters, for which the previous technique ${ }^{42}$ is less well suited.

\section{Chromophore-quadruple}

Force fields developed for the single chromophores and chromophore-pairs are presented in the SI. For the chromophore-triple (DPP-TT-T), our parameters are comparable with those published in the previous work by Do and Trosi, ${ }^{42}$ in terms of predicting the structural properties of this molecule. It is important to note that our parameters and those of Do and Trosi ${ }^{42}$ are obtained using two different procedures. In our work, the parameters are obtained using the fragmentation scheme, while those in Do and Troisi ${ }^{42}$ are obtained by optimising all parameters in the chromophore-triple in one go. The main problem in their approach is the cost in the computational time and thus we find it can be impractical for larger molecules, e.g. for the chromophore-quadruple in this instance. For molecular crystals, parameters for the chromophore-pairs DPP-T (which have not been published) are sufficient (for UBEQOK). However, for the polymer, parameters for the chromophore-quadruple (the monomer of the polymer) are required. The model shown in Figure 1a contains all information needed to construct the FFs for an oligomer of any arbitrary length, and parameters taken from the single chromophores and the chromophore-pairs are sufficient to obtain the parameters for the chromophore-quadruple. Parameters for the bonds, angles, and dihedrals that involved TC7 and TH0 atom types (Figure 1b) are not defined in the chromophorequadruple. Thus, these parameters are taken from the single chromophore TT, which is a reasonable approximation. In addition, sidechains are also treated as fragments and can be readily added if required. 


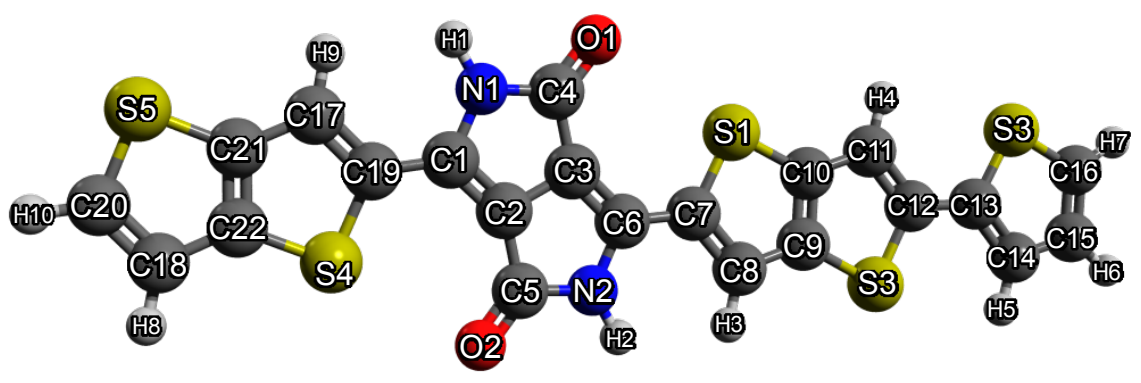

(a)

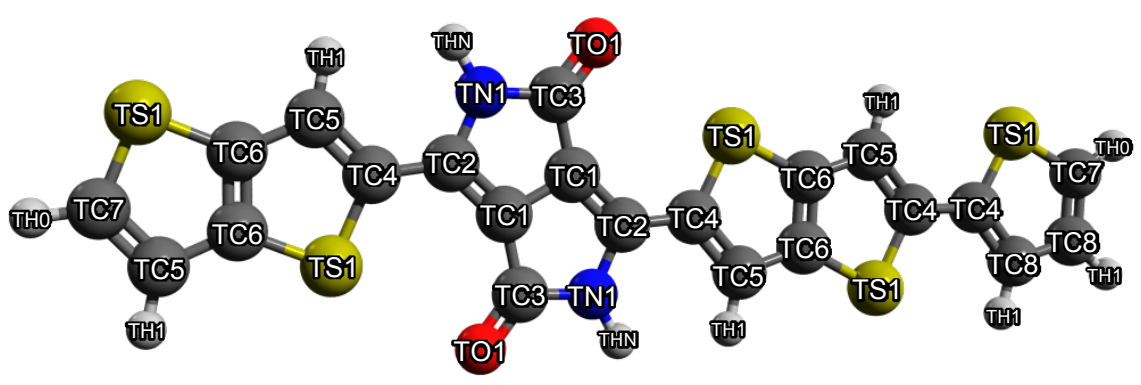

(b)

Figure 1: The structure of the TT-DPP-TT-T molecule: (a) are atom names and (b) are atom types.

The procedure for obtaining the parameters for the chromophore-quadruple is similar to that of the chromophore-pairs. In the first stage, all parameters apart from those of the "soft" dihedrals are obtained using the planar structures. In these geometries, the forces of the "soft" dihedrals are essentially zero. The initial "guessed" parameters are taken from the single chromophores. Missing parameters due to the combination of two chromophores (e.g. the bond parameters for $\mathrm{C} 6-\mathrm{C} 7$ or the angle parameters for $\mathrm{C} 6-\mathrm{C} 7-\mathrm{S} 1$ ) are taken from the chromophore-pairs. In the optimization process, it is possible to allow only the missing parameters to be optimized while the rest remain fixed at those obtained from the parameterization of the single chromophore. This approach is particular suitable for very large molecules with many parameters that require a long optimization time. For the case of the chromophore-quadruple (and also the chromophore-pair), it is possible to carry out the optimization processes for the whole molecule, and since the initial "guessed" parameters are reasonably good, it only required two iterations to converge. For chromophore-quadruple (and larger molecules e.g. oligomers), the "soft" dihedrals are not parameterized individually, 
and their parameters are taken directly from those in the chromophore-pairs. Figure 2 shows that the inclusion of the $\mathrm{T}$ ring slightly alters the torsion potential of the DPP-TT torsion. However, this effect is small enough to be safely neglected.

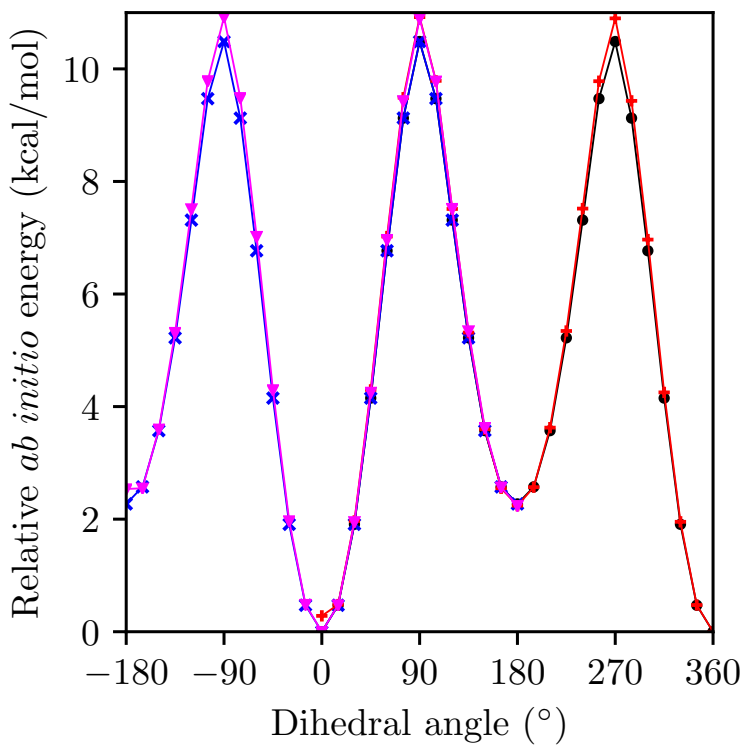

Figure 2: Comparison of the torsion potential of DPP-TT soft dihedral without the presence of chromophore $\mathrm{T}$ (black and blue lines) and with the presence of the chromophore $\mathrm{T}$ (red and purple lines). The scans are carried out using B3LYP $/ 6-31 \mathrm{G}^{*}$.

\section{Results and Discussion}

\section{Validation of the Parameters for the Chromophore-quadruple}

A comparison of the force and energy RMSD is shown in Table 1. Similar to the cases for single chromophores and chromophore-dimers (See tables S1 and S2 in the SI), AM1 gives the largest deviation for forces followed by $\mathrm{HF} / 6-31 \mathrm{G}^{*}$. B3LYP $/ 3-21 \mathrm{G}^{*}$ gives the smallest deviation from the reference method B3LYP/6-31G* for both forces and energies. The force RMSD is comparable to that of B3LYP $/ 3-21 \mathrm{G}^{*}$, while the energy RMSD is comparable to $\mathrm{HF} / 6-31 \mathrm{G}^{*}$. 
To demonstrate that our parameters are reliable for generating structures for quantum chem-

Table 1: Force and energy RMSD between calculations at B3LYP/6-31G* levels and the optimized FF or other levels of theory, for 100 arbitrary geometries of the chromophorequadruple TT-DPP-TT-T not used in the FF optimization process.

\begin{tabular}{|c|c|c|}
\hline Method & Force RMSD $\left(\mathrm{kcal} \mathrm{mol}^{-1} \AA^{-1}\right)$ & Energy RMSD $\left(\mathrm{kcal} \mathrm{mol}^{-1}\right)$ \\
\hline FF & 8.87 & 6.38 \\
AM1 & 22.45 & 5.22 \\
HF $/ 6-31 \mathrm{G}^{*}$ & 16.95 & 6.13 \\
B3LYP $/ 3-21 \mathrm{G}^{*}$ & 5.78 & 3.24 \\
\hline
\end{tabular}

istry calculations, we compare the electronic excited state properties of the chromophoretriple computed using trajectories from both classical and ab initio MD simulations. The distributions for the excitation energies and oscillator strengths are shown in Figure 3. These data show significant overlaps in both the transition energies and the oscillator strengths for the lowest two excited states $\mathrm{S}_{1}$ and $\mathrm{S}_{2}$, along the classical and ab initio trajectories. Similar trends are also observed for other transitions in this chromophore and also in other building blocks. Data for other chromophores can be found in the SI. Thus, these further suggest that the potetial energy surfaces generated by using our FFs are comparable to those generated using DFT. 

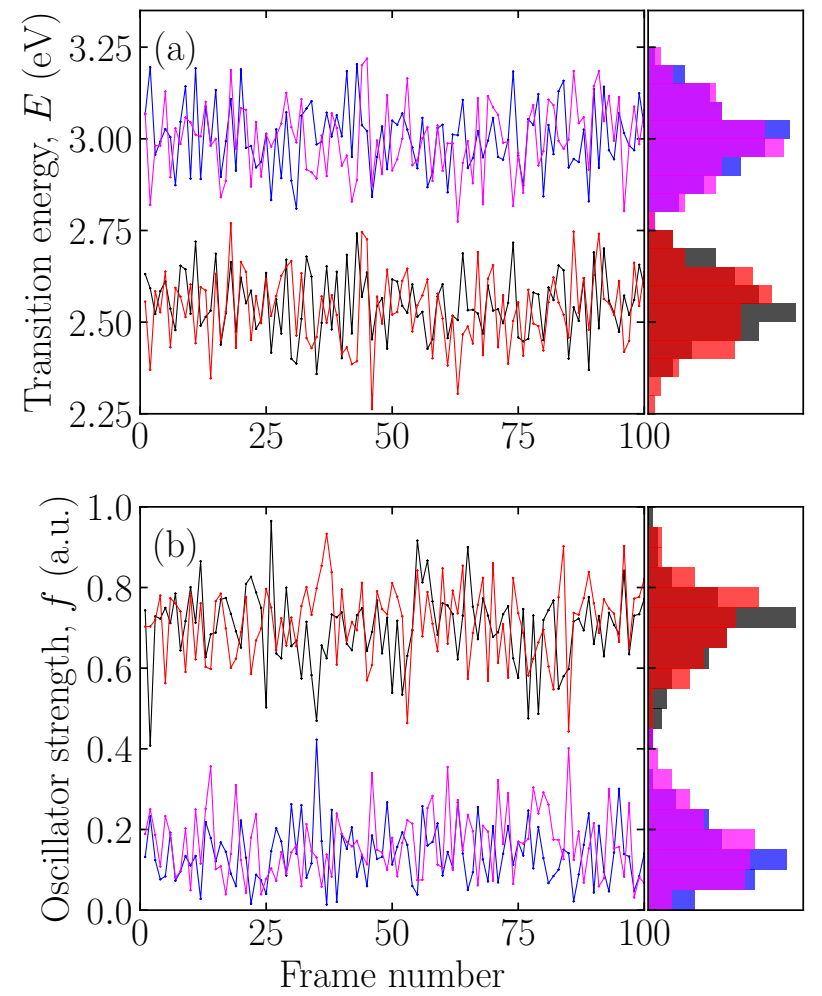

Figure 3: Comparison of the transition energies (a) and oscillator strengths $(f)$ (b) of the lowest two excited states computed using 100 uncorrelated frames taken from the classical (black and blue) and the $a b$ initio (red and magenta) trajectories for chromophore-triple (DPP-TT-T). All calculations are performed at the B3LYP/6-311G(d,p) level of theory.

Finally, Figure 4 compares the structural properties of the chromophore-quadruple computed for 100 snapshots along the classical and ab initio trajectories. As an example, we only show distributions for a bond, an angle and a soft dihedral of the chromophore in this figure. Other bonds, angles and dihedrals show the same trend. The good agreement observed for the structural properties in Figure 4 for the chromophore-quadruple underpin the agreement of the electronic excitation properties observed in Figure 3. Hence, this provides further confidence that our FFs can reproduce the ab initio potential energy surfaces, to which their parameters are fitted. 

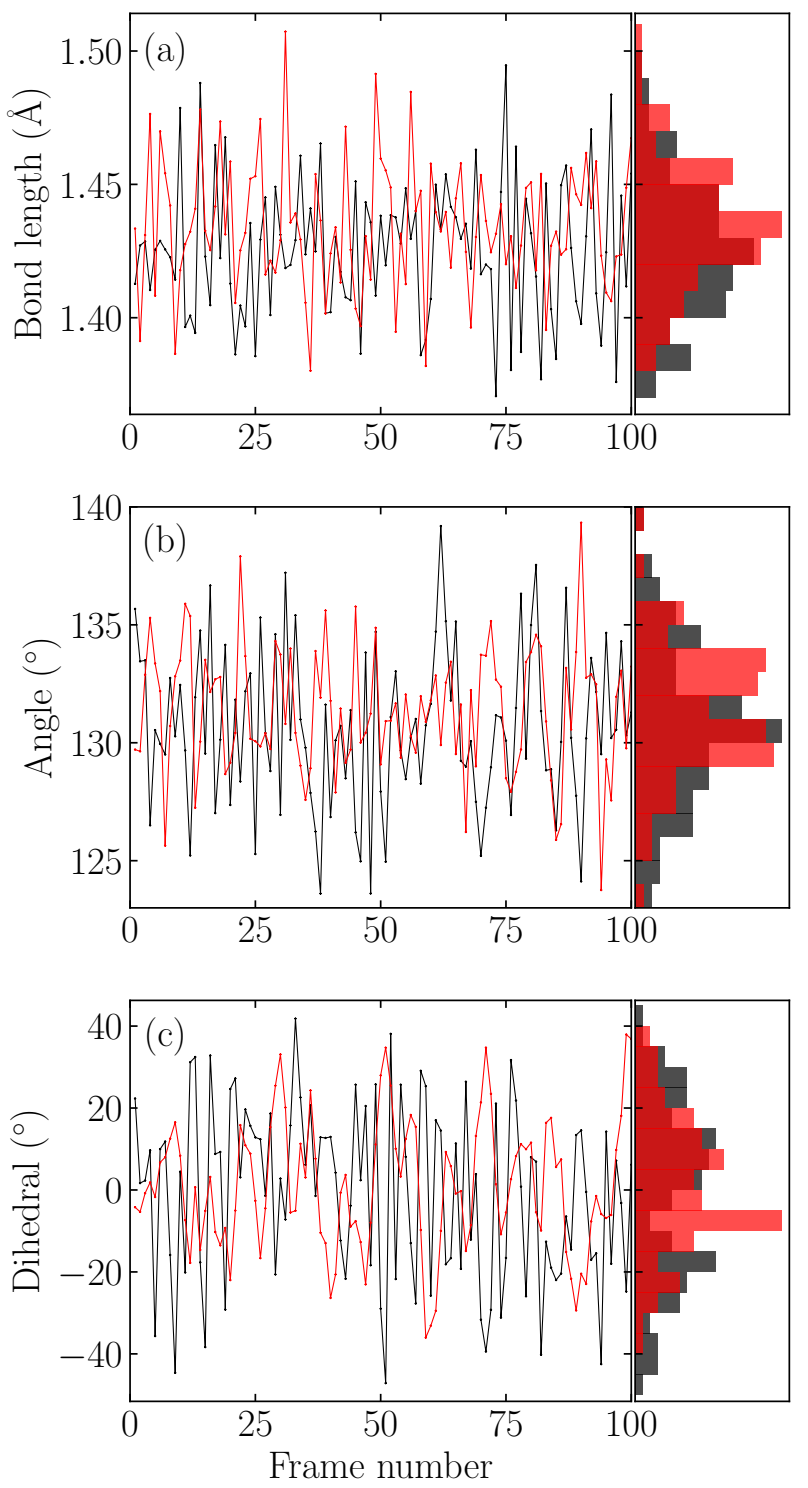

Figure 4: Comparison of the structural properties of chromophore-quadruple computed using 100 uncorrelated frames taken from the classical (black) and the ab initio (red) trajectories: (a) bond between $\mathrm{C} 6$ and $\mathrm{C} 7,(\mathrm{~b})$ angle between $\mathrm{C} 3, \mathrm{C} 6$ and $\mathrm{C} 7$, and (c) dihedral between C3, C6, C7 and S1.

\section{Conjugated Polymers}

\section{Molecular Dynamics Simulations}

MD simulations are carried out with NAMD $^{48}$ for 2040 -mers of the DPPTBT bulk conducting polymer. The monomer of DPPTBT is the chromophore-quadruple described in the 
previous section. Thus, the topology and parameters for DPPTBT oligomers are built upon the chromophore-quadruple. The polymer is initially placed into a cubic box of $109 \times 109 \times 109$ $\AA^{3}$ at a density of $0.5 \mathrm{~g} / \mathrm{cm}^{3}$ without overlaps. To achieve this, polymer chains are added into the box by growing segment by segment using the configurational bias Monte Carlo technique ${ }^{63}$ To avoid steric clashes, the growing process takes into account interaction with all atoms already positioned, whilst continuously monitoring the single chain conformations. The result of this procedure is that low energy sites are preferred over high energy sites. Thus, bulk disordered systems containing chain molecules in realistic equilibrium conformations are created.

Three-dimensional periodic boundary conditions are implemented. The system is minimized for 5000 steps and gradually heated to $300 \mathrm{~K}$ using 50 ps in the NVT ensemble. Then, the NPT ensemble at $1 \mathrm{~atm}$ and $300 \mathrm{~K}$ is employed for $200 \mathrm{~ns}$ to equilibrate the system. Finally, a further $100 \mathrm{~ns}$ is simulated in the NVT ensemble for calculating ensemble average properties. The Langevin thermostat ${ }^{64}$ with a damping coefficient equal to $1 \mathrm{ps}^{-1}$ is employed to keep the temperature constant. The period and decay parameters of the Langevin piston are set to 100 and 50, respectively, to maintain the pressure. A time step of $1 \mathrm{fs}$ for integration of the equations of motion is used throughout the simulation. A cutoff of $12 \AA$ is used for nonbonded interactions. The particle mesh Ewald algorithm ${ }^{65}$ is used to calculate long-range electrostatic interactions. Figure 5 gives a visualization of the system and Figure 6 shows the density variation with respect to the simulation time. Starting from $0.5 \mathrm{~g} / \mathrm{cm}^{3}$, the system reaches about $1 \mathrm{~g} / \mathrm{cm}^{3}$ after $100 \mathrm{~ns}$, and eventually converges to about 1.035 $\mathrm{g} / \mathrm{cm}^{3}$. A density of around $1 \mathrm{~g} / \mathrm{cm}^{3}$ is a typical value for conducting polymers. The same final density is also observed for different starting densities and different chain lengths.

First of all, we inspect the radius of gyration of the polymer under ambient conditions. The range of gyration radii (Figure S28) is between 125 to $160 \AA$, which indicates these 


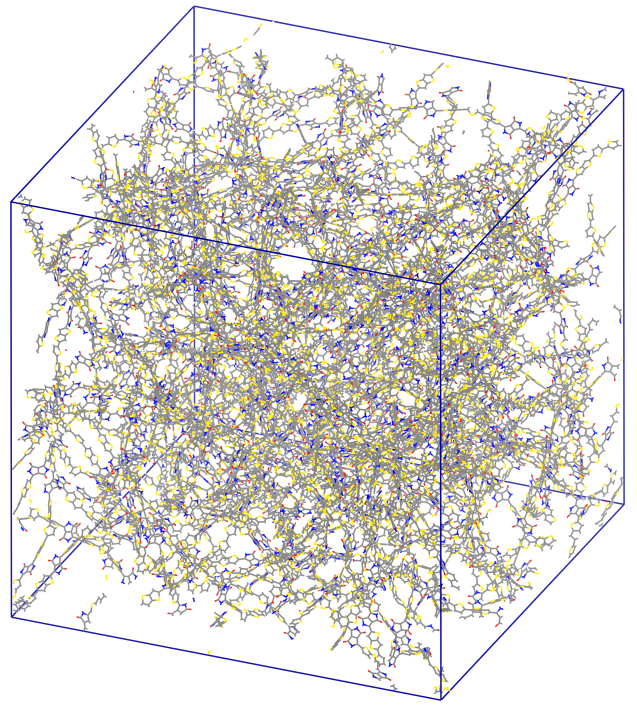

(a)

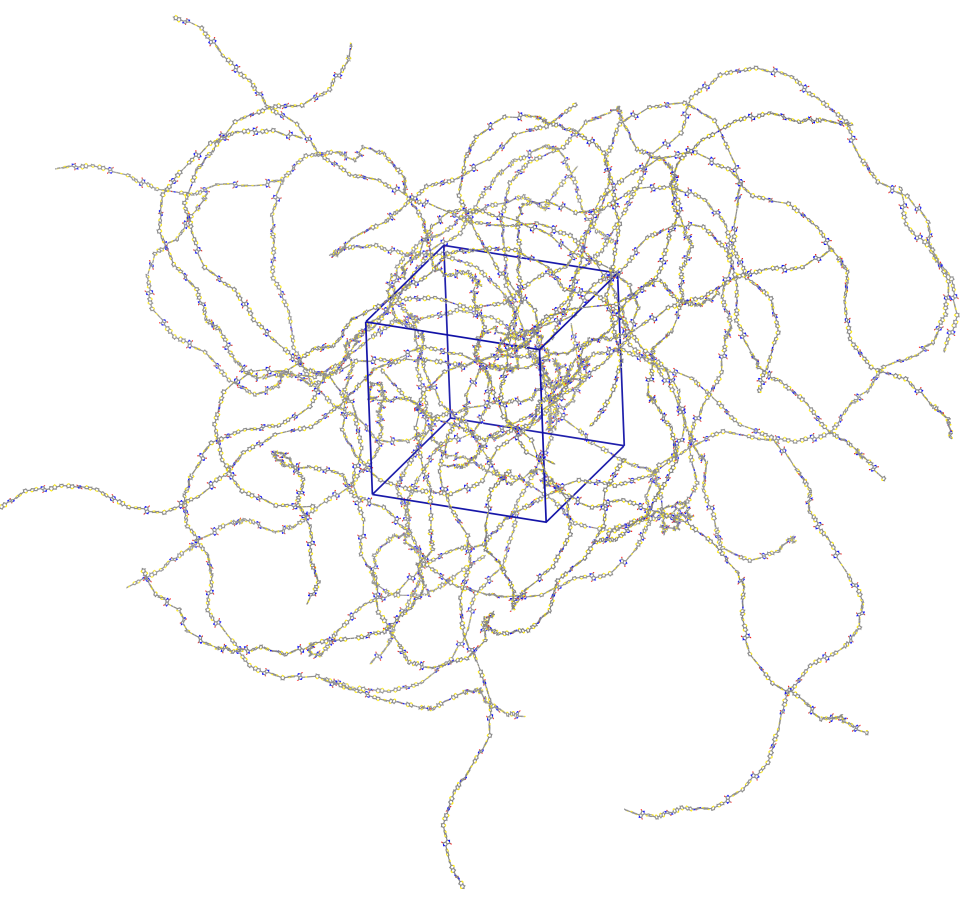

(b)

Figure 5: DPPTBT amorphous cell: 40 monomers/chain, 20 chains, density $0.5 \mathrm{~g} / \mathrm{cm}^{3}$. (a) In-cell display style. (b) Out-cell display style.

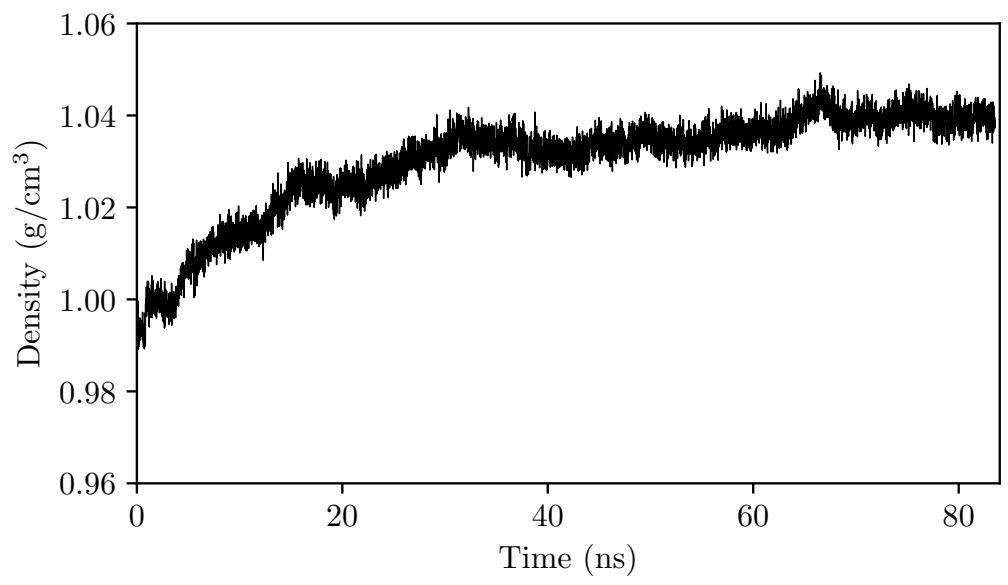

Figure 6: Time evolution of the density in the last $100 \mathrm{~ns}$ of the $200 \mathrm{~ns}$ NPT ensemble simulation.

chains are rather elongated. This property of the polymers very quickly reaches equilibrium and remains essentially unchanged during the course of the simulations at $300 \mathrm{~K}$. This behaviour has been observed previously for other conducting polymers. ${ }^{7}$ 
In general, the flexible dihedral angles play an important role in the electronic properties of conducting polymers. A planar configuration leads to high hole mobility, due to the formation of the $\pi$ orbitals along the backbone. ${ }^{66}$ To examine the planarity of this polymer, we compute the distribution of the soft dihedrals along the chain backbone. Figure 7 shows the distribution between DPP and TT of the first monomer in chain one. A strong peak near $0^{\circ}$ is observed. This distribution is also found for the torsion DPP-TT along chain number one and in other chains. Similarly, for the distribution of the torsions between TT and $\mathrm{T}$ and between DPP and $\mathrm{T}$, we also observe peaks around $180^{\circ}$ or $0^{\circ}$. This suggests that dihedral angles near $0^{\circ}$ and $180^{\circ}$ are favoured thermodynamically, which indicates planar configurations for DPPTBT at the temperature and density studied.

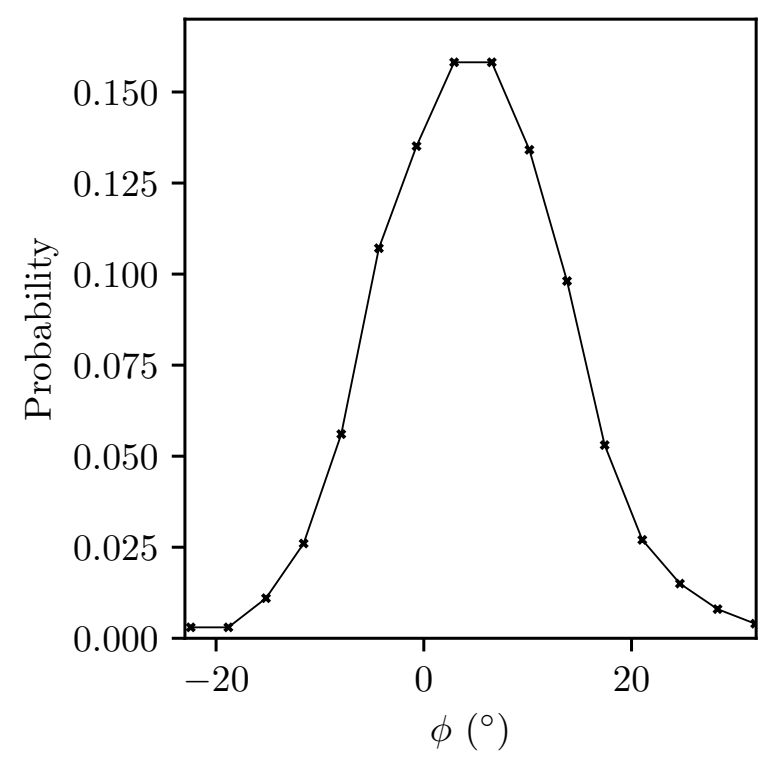

Figure 7: Distribution of dihedrals of polymer during 100 ns NVT simulation.

Figure 8 shows the RDFs of all N1, S1 and O1 atoms in the system, respectively. These RDFs give quantitative information about the separation (or packing) of surrounding atoms from any given atom. There are three distinctive peaks observed for all three atom types at 
roughly 4,8 and $20 \AA$. These are the radii for which other atoms most likely to be found. The first two peaks in the RDF for sulfur atoms (Figure 8b) are similar to that observed for the one chain simulations of the PCDTBT conducting polymer, where sulfur peaks at about 5 and $9 \AA$ were observed. ${ }^{66}$ Thus, the first two peaks at short distances in our RDFs can be associated with the distributions of atoms along the chain (i.e. intra-chain packing) and the last peak (at about $20 \AA$ ) gives the inter-chain distributions. 

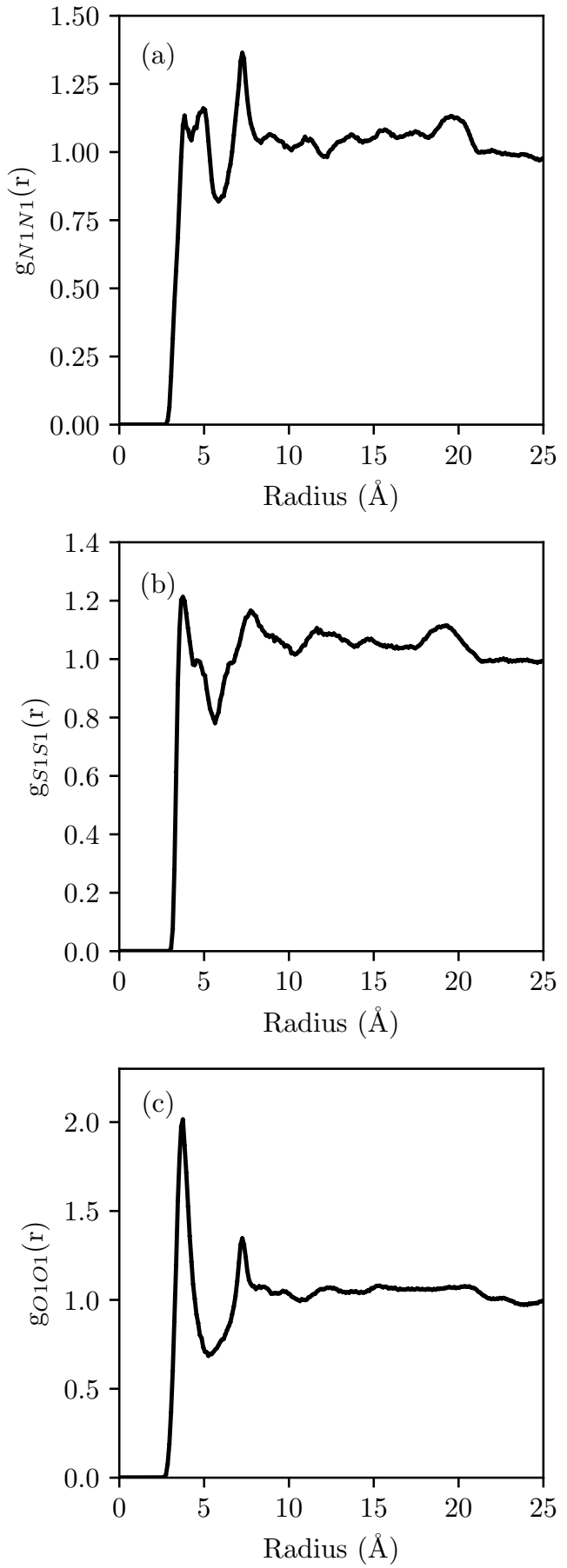

Figure 8: Radial distribution function (RDF) for given atoms in the polymer. (a) N1, (b) $\mathrm{S} 1$ and (c) $\mathrm{O} 1$. 


\section{Electronic Structure Properties}

To gain insights into the electronic structure of the polymer, we first study the bulk density of states (DOS). Figure 9 shows the bulk DOS for the system of 20 chains of 40-mers averaged over ten snapshots from 10 to 100 ns each separated by 10 ns. Here, the background charges are not included to save the computational time, due to the effect of electrostatic disorder, which is relatively weak for semiconducting polymer. ${ }^{7}$ The long-range corrected functional $\omega \mathrm{B} 97 \mathrm{X}-\mathrm{D}$ is employed along with the $3-21 \mathrm{G}^{*}$ basis set for this task. We found that the fluctuations in the DOS per chain per monomer for this system are almost negligible. Similarly, the DOS does not vary significantly with the chain lengths between 20 to 40 monomers, which agrees with a similar study on amorphous MEH-PPV polymers. ${ }^{7}$ Calculations (only for one snapshot) using a larger basis set, i.e. 6-31G*, indicate that the discrepancy in the computed DOS between the two basis sets is negligible, especially around the HOMO-LUMO gap. Thus, it is sufficient to employ the smaller basis set $\left(3-21 \mathrm{G}^{*}\right)$ for characterising the electronic excitation in this type of conducting polymer using TDDFT.

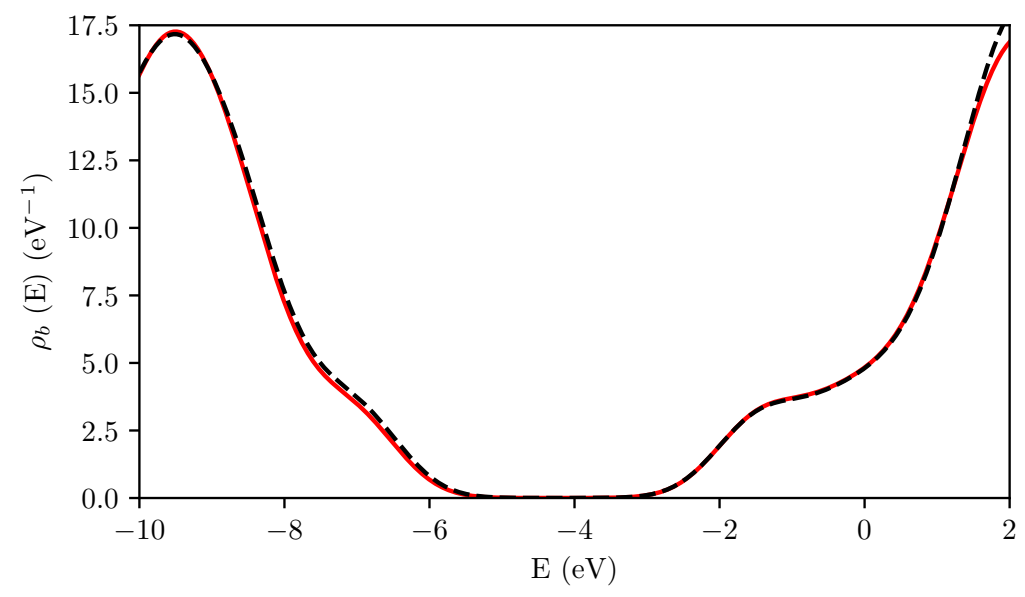

Figure 9: Bulk density of states computed for the 40-mer chains using the long-range corrected functional $\omega$ B97X-D. Solid red line is the result using $3-21 \mathrm{G}^{*}$ basis set averaged over 10 snapshots from 10 to $100 \mathrm{~ns}$ separated by $10 \mathrm{~ns}$. Dashed black line is the result using $6-31 \mathrm{G}^{*}$ basis set for a single chain at $100 \mathrm{~ns}$.

To investigate the nature of the electronic excitation of the polymer, we analyse the e-h 
distributions and the transition densities for the lowest three singlet excitations of a single chain. Among several other descriptors, the $\Lambda$-index proposed by Peach et al. ${ }^{54}$ has gained much attention, and has been widely employed as a diagnostic tool for monitoring TDDFT results. ${ }^{67}$ This method is based on the overlap of molecular orbital moduli. However, in some difficult cases, this index may not be able to locate problematic excitations. ${ }^{68}$ To provide a robust tool for exploring the excited state metric, another index called the $\Delta \mathrm{r}$-index has been introduced. ${ }^{55}$ This index is based on the charge centroids of the orbitals involved in the transition. It is easy to interpret, because it measures the average e-h distance upon excitation. The $\Delta$ r-index works particularly well for the CT excitations for which reliable charge transfer length can be obtained.

To characterise the electronic excitation of the conducting polymer, we computed all the descriptors discussed previously and we present the results for the three lowest singlet excitations for the 40-mer chain in Table 2. All TDDFT calculations are performed at the $\omega \mathrm{B} 97 \mathrm{X}-\mathrm{D} / 3-21 \mathrm{G}^{*}$ level of theory. Table 2 shows that the $\mathrm{S}_{0} \rightarrow \mathrm{S}_{1}$ is clearly a local excitation with a relatively small $\mathrm{D}$-index and $\Delta \mathrm{r}$-index, which indicate that the hole and electron are quite close. This is also confirmed by, for example, the high value in the exciton binding energy and the overlap $\mathrm{S}_{\mathrm{r}}$-index. Further investigation of this transition using the contributions of the molecular orbitals of atoms and chromophores in the polymer chain to the e-h (Figure 10) shows that both the hole and electron originate from monomer number 30 and the e-h also resides on this monomer (mainly on the DPP chromophore). This is confirmed by a plot of the fragment transition density matrix (Figure 11), which shows the distribution of charges along the polymer chain. According to the heat map shown in Figure 11, we can also observe that the electron and hole are mainly distributed on monomer 30 . Since there is no clear off-diagonal element, this transition does not cause significant electron transfer between monomers. Although there are some small fractions of charge on the nearest neighbours, as can be observed from Figures 10 and 11, the main character of this excitation is 
its localization on monomer 30 .

Table 2: Electronic excitation indices for the three lowest singlet excited states.

\begin{tabular}{|l|c|c|c|c|c|c|c|c|}
\hline Transition & $D(\AA)$ & $S_{r}$ (a.u.) & $H(\AA)$ & $t(\AA)$ & $\delta \sigma(\AA)$ & $E_{\text {coul }}(\mathrm{eV})$ & $\Lambda$-index & $\Delta$ r-index \\
\hline $\mathrm{S}_{0} \rightarrow \mathrm{S}_{1}$ & 0.55 & 0.91 & 7.70 & -4.37 & -0.48 & 2.03 & 0.81 & 2.40 \\
\hline $\mathrm{S}_{1} \rightarrow \mathrm{S}_{2}$ & 7.45 & 0.72 & 20.26 & -8.31 & -3.13 & 0.45 & 0.62 & 16.75 \\
\hline $\mathrm{S}_{2} \rightarrow \mathrm{S}_{3}$ & 9.93 & 0.48 & 19.73 & -5.84 & 0.22 & 0.27 & 0.59 & 25.34 \\
\hline
\end{tabular}

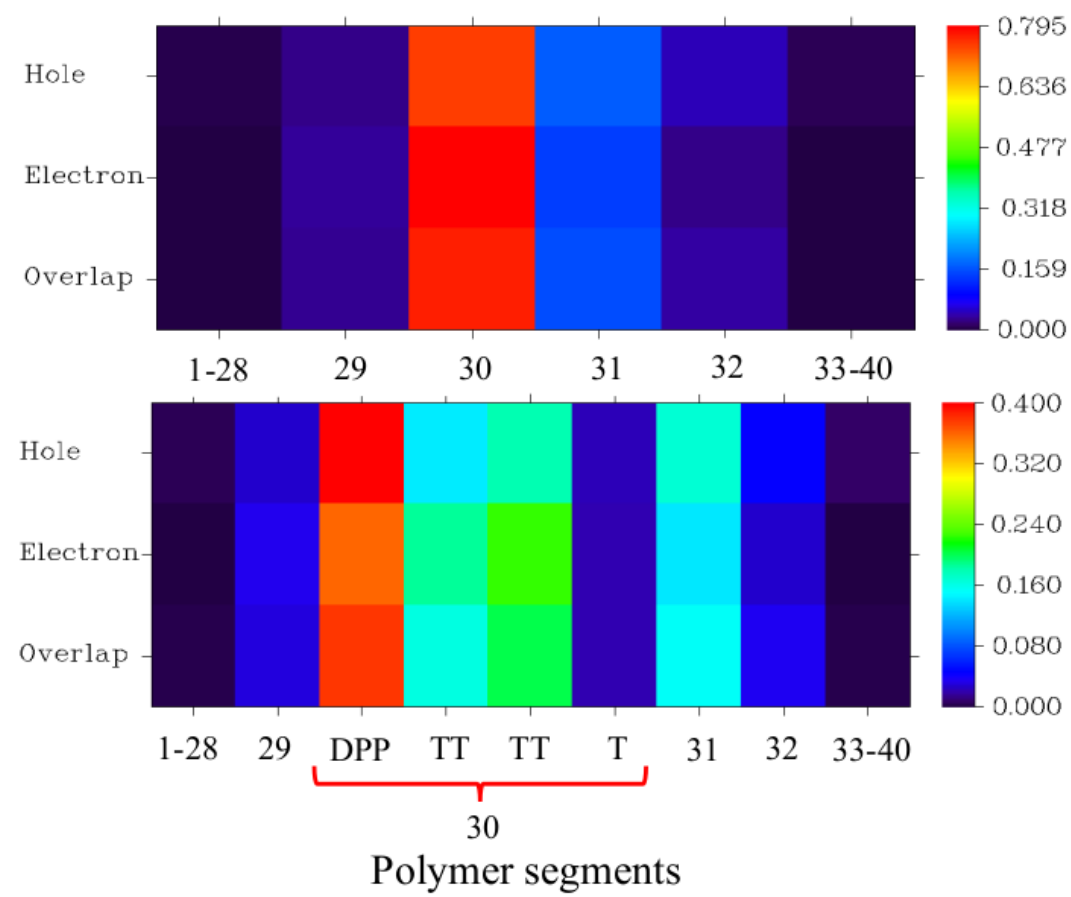

Figure 10: Heat map of fragment contributions (percentage contribute) to hole and electron for the $\mathrm{S}_{0} \rightarrow \mathrm{S}_{1}$ transition. The numeric ticks in the x-axis denote the monomer sequence while the letters indicate the chromophore within the monomers. 


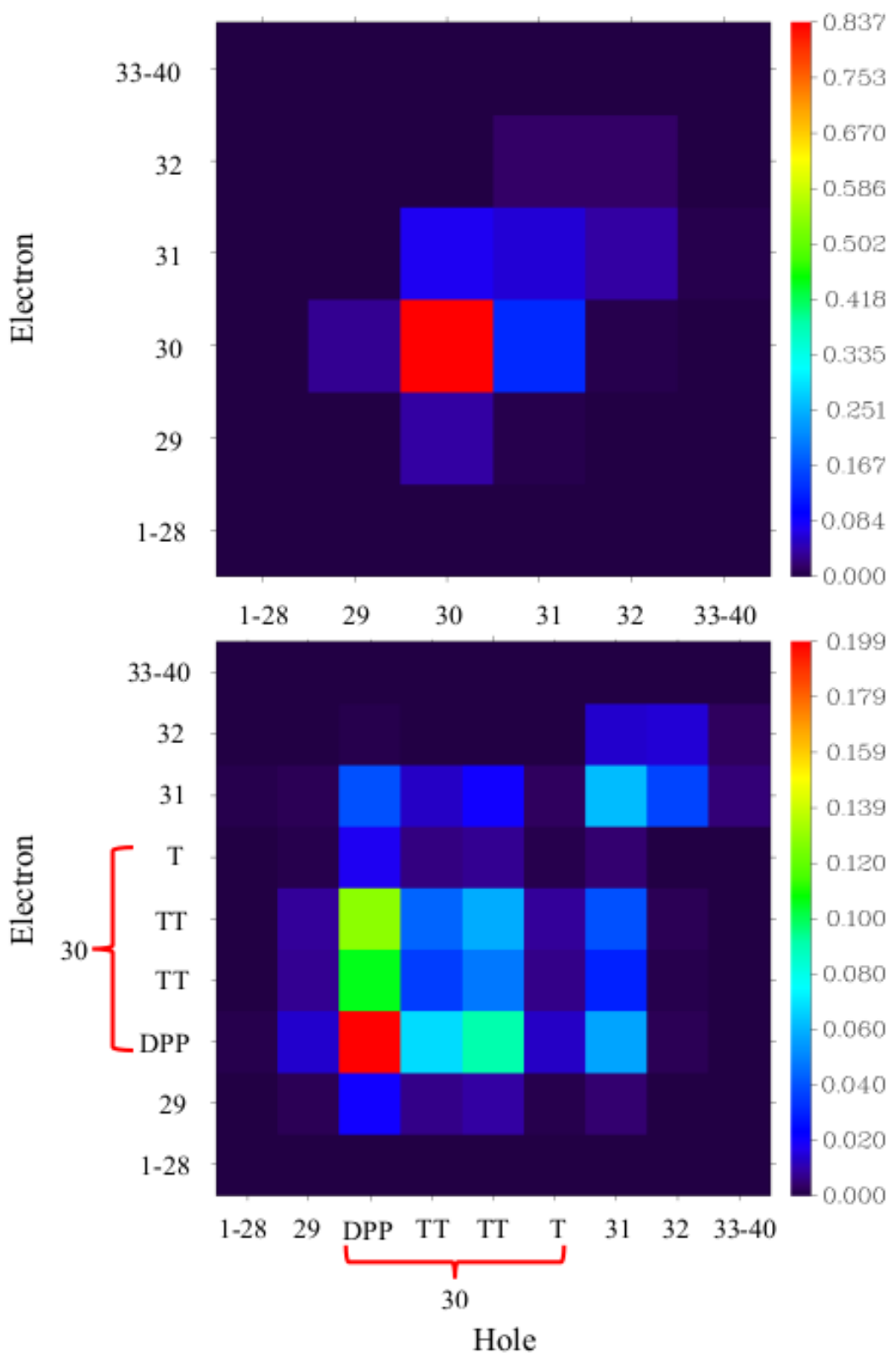

Figure 11: Heat map of fragment transition density matrix for the $\mathrm{S}_{0} \rightarrow \mathrm{S}_{1}$ transition. The numeric ticks in the $\mathrm{x}$-axis denote the monomer sequence while the letters indicate the chromophore within the monomers.

From Table 2, we can see that the CT character for the $\mathrm{S}_{0} \rightarrow \mathrm{S}_{2}$ and $\mathrm{S}_{0} \rightarrow \mathrm{S}_{3}$ transitions is quite strong with much larger $\mathrm{D}$ - and $\Delta$ r-indices and substantial smaller exciton binding energies than the $\mathrm{S}_{0} \rightarrow \mathrm{S}_{1}$ transition. In addition, the average degree of spatial extension of the hole and electron distribution (H-index) is also greater for the latter two excitations. Thus, the $\mathrm{S}_{0} \rightarrow \mathrm{S}_{2}$ and $\mathrm{S}_{0} \rightarrow \mathrm{S}_{3}$ transitions are delocalised CT states. The degree of delocalisation 
can be observed from the e-h distribution and the transition density matrix plots, which are shown in Figures 12 and S31 for the $\mathrm{S}_{0} \rightarrow \mathrm{S}_{2}$ transition and Figures S32 and S33 in the SI for $\mathrm{S}_{0} \rightarrow \mathrm{S}_{3}$. For example, in the $\mathrm{S}_{0} \rightarrow \mathrm{S}_{2}$ transition, the electron and hole are mainly contributed to by monomers 13, 14 and 15 and the overlap is centred around monomer 14 . However, contributions to both electron and hole from other monomers away from the three central monomers are also observed. The conjugation length (delocalisation length) is approximately four to six monomers based on the analysis of the e-h distributions and the transition density matrices for both CT states. This is in good agreement with other theoretical and experimental studies for amorphous conducting polymers. ${ }^{7,18,69}$ Calculations for another 40 -mer chain also show a similar trend for the first three lowest singlet excitations, which indicate that the two chains may share some similarities in their geometries. As the main focus of this work is on force field development, a thorough investigation of the relationship between the structures and electronic excitation properties of this class of conducting polymers will be carried out in future work. 


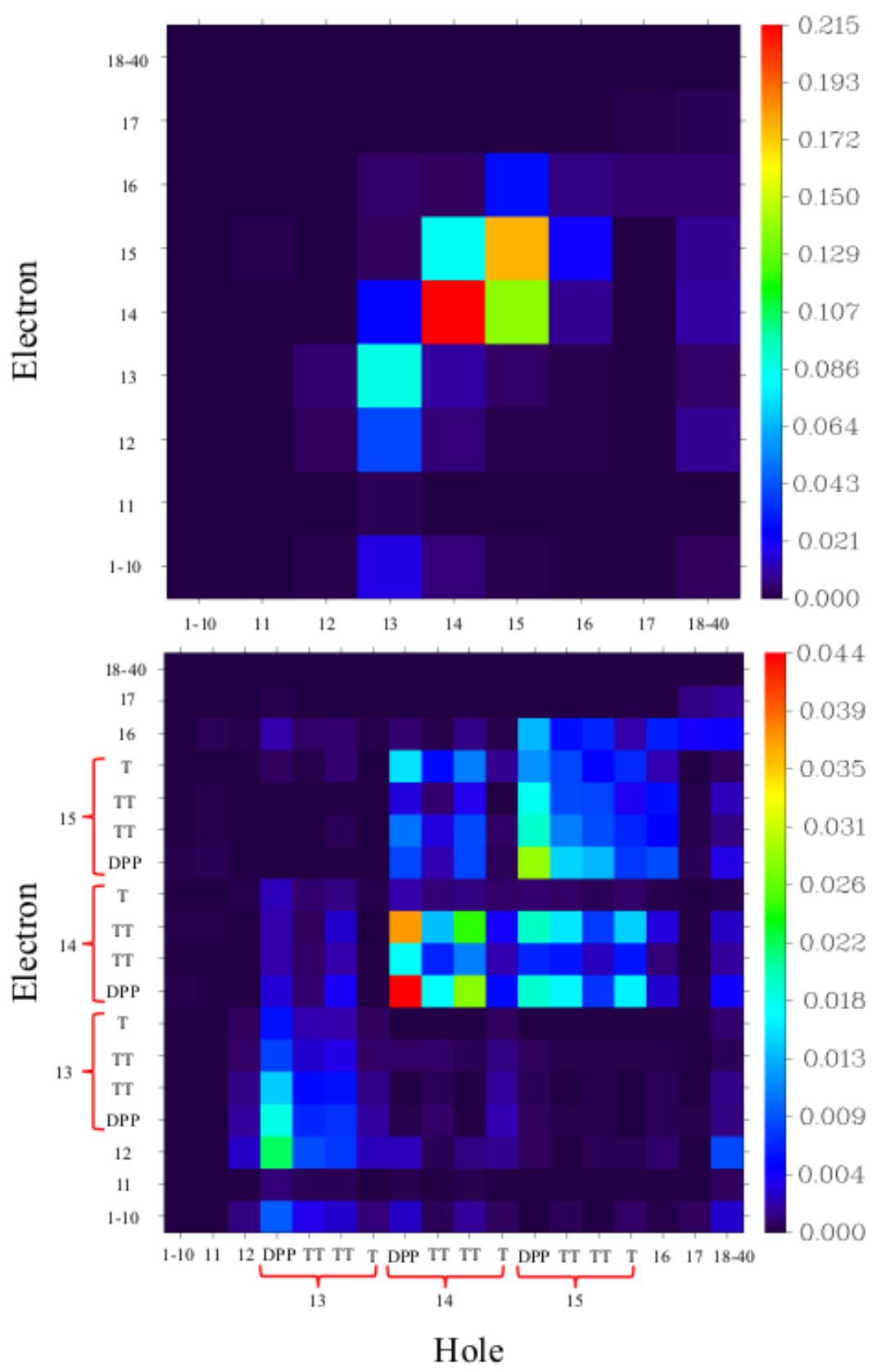

Figure 12: Heat map of fragment transition density matrix for the $S_{0} \rightarrow S_{2}$ transition. The numeric ticks in the $\mathrm{x}$-axis denote the monomer sequence while the letters indicate the chromophore within the monomers. 


\section{Molecular Crystals}

\section{Molecular Dynamics Simulations}

As another example, we study the dynamics of the molecular crystal UBEQOK, which has

been synthesised recently (Figure S34). ${ }^{59}$ The equilibrium structures generated from MD simulations will be valuable for understanding the exciton dynamics in this new material. First of all, a $3 \times 8 \times 2$ supercell, which comprises 192 molecules is created. Then, the system is minimized using 5000 steepest descent steps followed by 0.5 ns heating in the NPT ensemble (at $300 \mathrm{~K}$ and $1 \mathrm{~atm}$ ). It is equilibriated for another $100 \mathrm{~ns}$ and continued for another $10 \mathrm{~ns}$ for production. All other simulation parameters are kept the same as those in the simulations of the polymeric system.

Since the simulations are performed under ambient conditions, it is expected that the molecules do not move very far from the original crystal structure and this can be observed from the root mean squared displacement of all molecules in the system (see Figure S27 in the SI). In other words, there is no melting and the molecules vibrate about their lattice points. Further insight into the microscopic structure of molecular crystal UBEQOK can be obtained from the distribution of torsional angles (Figure S30). The peak around $0^{\circ}$ confirms that the molecular crystal UBEQOK's backbone is planar.

To gain insights into the nature of the fluctuation of the excitonic coupling between neighboring molecules in UBEQOK molecular crystal due to thermal motions, the magnitude and time scale of the fluctuation of the excitonic coupling are investigated using a combined QM and MD approach. To compute the excitonic couplings for a pair of molecules, we employ the general diabatization scheme proposed by Arago and Troisi, ${ }^{70}$ which is capable of taking into account both short- and long-range effects. The short-range excitonic coupling contribution is inherent in molecular crystals and aggregates and is responsible for 
the fluctuation of the excitonic coupling in the presence of the nuclear thermal motions. ${ }^{70}$ Here, only the couplings of nearest neighbors are considered, as they have been shown to be adequate to describe quantitatively the excited state properties of molecular crystals. ${ }^{71}$ In addition, the coupling between molecular pairs at longer distances is dominated by the electrostatic term, which is not sensitive to small thermal fluctuations. ${ }^{70}$

Two types of dimers with closest intermolecular contact for the exciton transport in UBQOK molecular crystal are identified. Figure 13 shows the arrangement of these two structures with structure labelled (A) the parallel $\pi$-stacked columns where the intermolecular distance is around $5.7 \AA$ and (B) a tilted structure. To construct the time evolution of the excitonic coupling in UBEQOK, nearest neighbors are extracted from 1000 equilibrium MD snapshots each separated by $50 \mathrm{fs}$. Then, the excitonic couplings for dimers (A) and (B) are computed using TDDFT at the $\omega$ B97X-D/6-31G* level of theory.

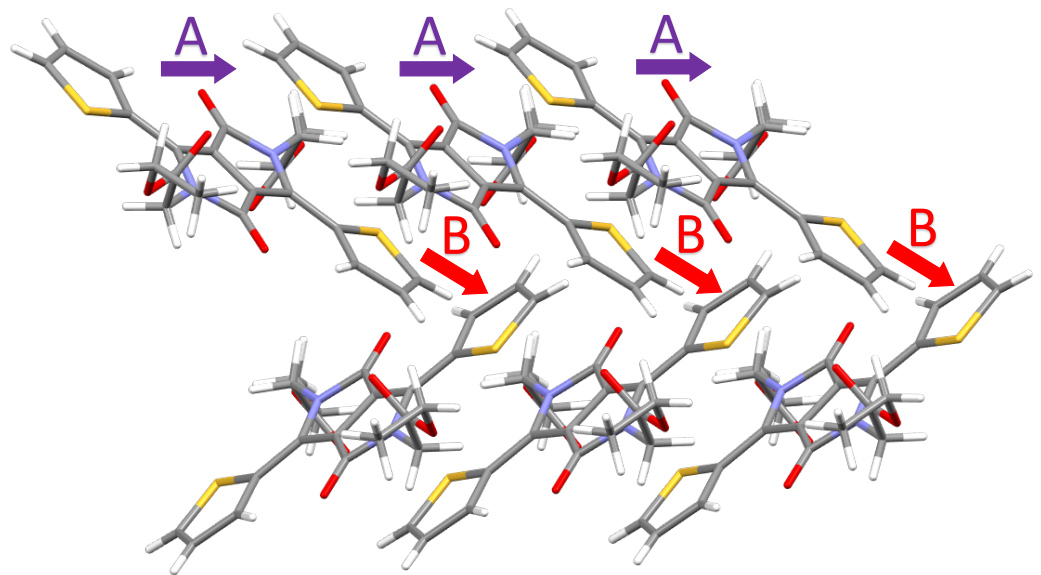

Figure 13: Example of crystal packing of the UBEQOK showing the most relevant dimers for the exciton transport: Parallel (A) and tilted (B) structures. 

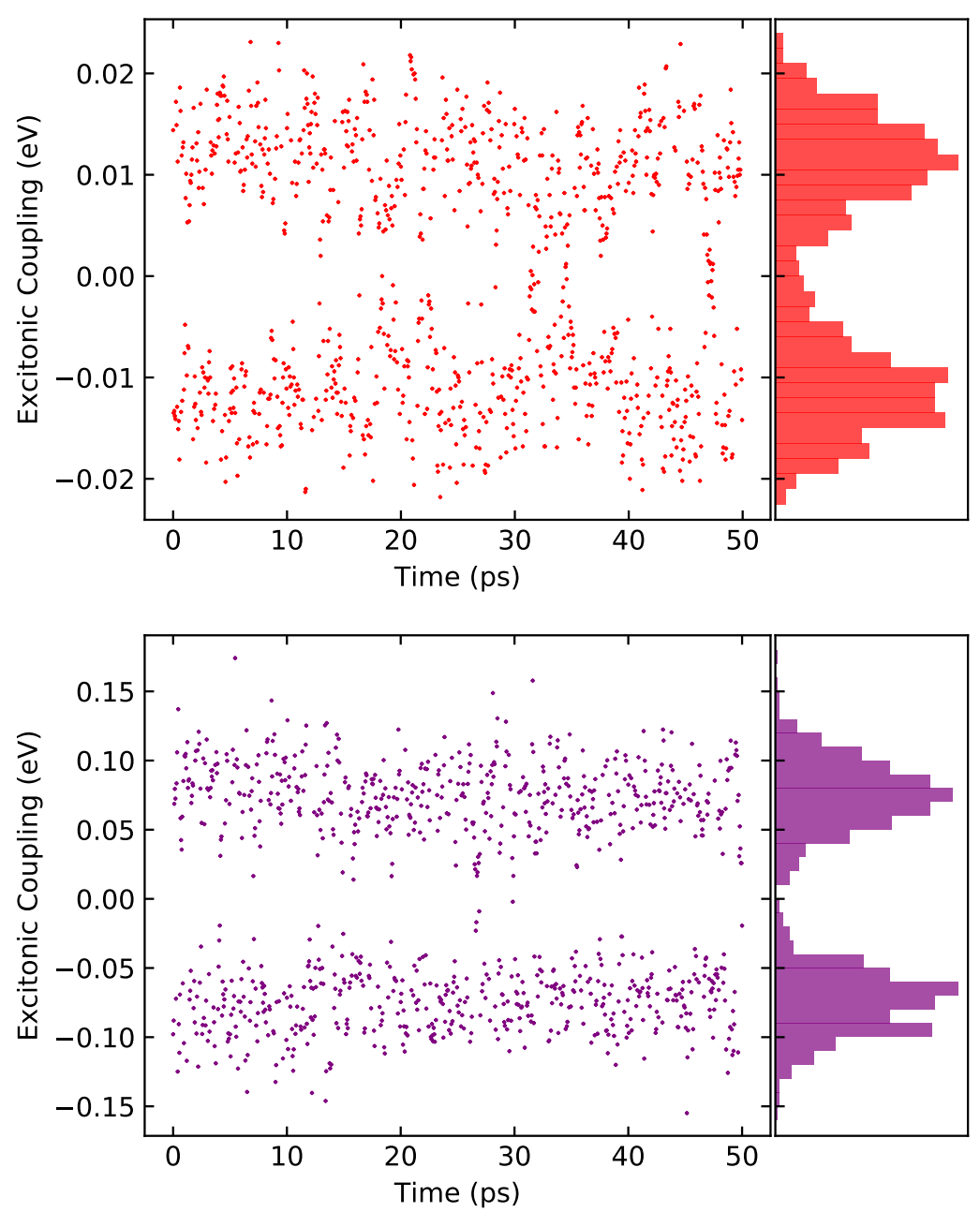

Figure 14: Time evolution and distribution of the excitonic couplings computed at $300 \mathrm{~K}$ for dimer A (bottom panel) and B (top panel).

Figure 14 shows the time evolution and the distribution of the excitonic couplings computed for the two UBEQOK dimers (A) and (B). The excitonic couplings are bimodal distributions with each one being normally distributed (positive- and negative-coupling distribution). Both distributions of coupling values have approximately the same absolute mean and the probability of having a positive or a negative coupling is equally likely. The formation of a bimodal distribution is due to the flexibility between DPP and T chromophores, which causes the change in the signs of the transition dipole moments. Figure 14 shows that the magnitude of the couplings for UBEQOK falls into the same range as those computed for other molecular crystals e.g. DCVSN5 ${ }^{72}$ and $\mathrm{H}_{2}-\mathrm{OBP}_{\mathrm{c}} \cdot{ }^{73}$ In addition, the largest excitonic 
coupling is also observed for the parallel structure for this system.

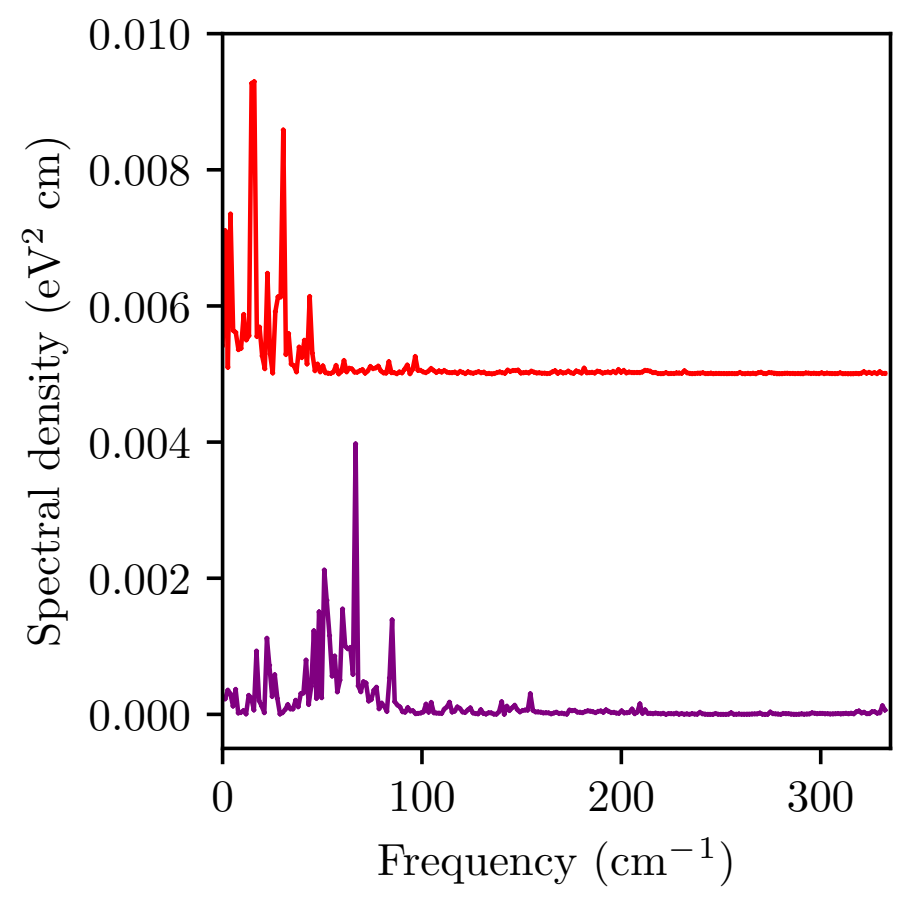

Figure 15: Fourier transformation of the autocorrelation function of the excitonic couplings computed at $300 \mathrm{~K}$ for the dimers A (purple) and B (red). The spectral density for dimer B is shifted upward by $0.003 \mathrm{eV}^{2} \mathrm{~cm}$.

To investigate the characteristic timescale of the fluctuation in the excitonic couplings, Fourier transforms of the autocorrelation functions $\langle\delta J(0) \delta J(t)\rangle$, where $J(t)$ is the coupling as function of time, are computed for both positive- (Figure 15) and negative-coupling distributions (Figure S35 in the SI). Here, the deviation from the average excitonic coupling is defined as $\delta J(t)=J(t)-\langle J(t)\rangle$. Figures 15 and S35 show that low frequency vibrations below $100 \mathrm{~cm}^{-1}$ are observed for both dimers (A) and (B), which is in good agreement with previous studies on other molecular crystals. ${ }^{70,72,73}$ These low frequency vibrations are believed to be responsible for the large modulation of the excitonic coupling. 


\section{Conclusion and Outlook}

A robust approach for generating accurate FFs for conjugated systems has been applied and extended for applications where subsequent quantum chemical calculations are employed. Built upon the force-matching technique, the method provides a consistency between the potential energy surface generated by empirical FFs and that generated by any ab initio method of choice. The approach is particularly suitable for applications that involve excited state calculations where accurate FFs to describe the excited state potential energy surface are needed. As an example, we parametrize the FFs and study the dynamics as well as the electronic excited properties of conjugated conducting polymers and molecular crystals containing diketopyrrolopyrrole, thiophene, and thieno[3,2-b]thiophene. The good agreement observed for both the structural and electronic excitation properties for all building block chromophores provides confidence that our FFs can reproduce the ab initio potential energy surfaces, for which their parameters are fitted to.

In this work, we employ OPLS FFs and the DFT at the B3LYP/6-31G* level of theory to illustrate how the desired optimised parameters can be obtained. However, the procedure is general and can be applied to any other combination of FF functional form and electronic structure method. The force RMSD between ab initio and empirical FFs can be improved by including more fitting parameters, such as introducing more atom types, including coupling between degrees of freedom and anharmonicity, or modifying the FF functional form. Our optimised FFs, by construction, are not transferable by atom types. However, the conjugated core such as DPP, TT, T, DPP-TT, DPP-T and TT-T could be treated as transferable. FFs for larger molecules can be constructed from these basic units, and thus allow access to the dynamics of conjugated macro-assemblies. 


\section{Supporting Information}

A brief description of the OPLS force field; discussion on choosing different atom types; force field development for single chromophores and chromophore pairs; additional properties of the conducting polymer and molecular crystal; force field parameters. This information is available free of charge via the Internet at http://pubs.acs.org

\section{Acknowledgements}

We are grateful for access to the University of Nottingham High Performance Computing Facility both in Ningbo China and Nottingham. Ling Jiang thanks the Faculty of Science and Engineering at University of Nottingham Ningbo China for a PhD scholarship. We thank Professor David Amabilino for fruitful discussions and critical comments on the manuscript. This work is financially supported by the National Natural Science of China (Grant No. 21850410456) and the University of Nottingham Propulsion Futures Beacon.

\section{References}

(1) Rivnay, J.; Mannsfeld, S. C.; Miller, C. E.; Salleo, A.; Toney, M. F. Quantitative determination of organic semiconductor microstructure from the molecular to device scale. Chem. Rev. 2012, 112, 5488-5519.

(2) Brunner, K.; Van Dijken, A.; Börner, H.; Bastiaansen, J. J. A. M.; Kiggen, N. M. M.; Langeveld, B. M. W. Carbazole Compounds as Host Materials for Triplet Emitters in Organic Light-Emitting Diodes: Tuning the HOMO Level without Influencing the Triplet Energy in Small Molecules. J. Am. Chem. Soc. 2004, 126, 6035-6042.

(3) Huang, D. M.; Mauger, S. A.; Friedrich, S.; George, S. J.; Dumitriu-LaGrange, D.; 
Yoon, S.; Moulé, A. J. The consequences of interface mixing on organic photovoltaic device characteristics. Adv. Funct. Mater. 2011, 21, 1657-1665.

(4) Lei, T.; Cao, Y.; Fan, Y.; Liu, C. J.; Yuan, S. C.; Pei, J. High-performance air-stable organic field-effect transistors: Isoindigo-based conjugated polymers. J. Am. Chem. Soc. 2011, 133, 6099-6101.

(5) Bronstein, H. et al. Thieno[3,2- b]thiophene-diketopyrrolopyrrole-containing polymers for high-performance organic field-effect transistors and organic photovoltaic devices. J. Am. Chem. Soc. 2011, 133, 3272-3275.

(6) Noriega, R.; Rivnay, J.; Vandewal, K.; Koch, F. P.; Stingelin, N.; Smith, P.; Toney, M. F.; Salleo, A. A general relationship between disorder, aggregation and charge transport in conjugated polymers. Nat. Mater. 2013, 12, 1038-1044.

(7) Qin, T.; Troisi, A. Relation between Structure and Electronic Properties of Amorphous MEH-PPV Polymers. J. Am. Chem. Soc. 2013, 135, 11247-11256.

(8) Kline, R. J.; McGehee, M. D. Morphology and charge transport in conjugated polymers. Polym Rev 2006, 46, 27-45.

(9) Guo, X.; Baumgarten, M.; Müllen, K. Designing $\pi$-conjugated polymers for organic electronics. Prog. Polym. Sci. 2013, 38, 1832-1908.

(10) Alberga, D.; Mangiatordi, G. F.; Torsi, L.; Lattanzi, G. Effects of annealing and residual solvents on amorphous P3HT and PBTTT films. J. Phys. Chem. C 2014, 118, 86418655.

(11) Marsh, H. S.; Jankowski, E.; Jayaraman, A. Controlling the morphology of model conjugated thiophene oligomers through alkyl side chain length, placement, and interactions. Macromolecules 2014, 47, 2736-2747. 
(12) Mollinger, S. A.; Krajina, B. A.; Noriega, R.; Salleo, A.; Spakowitz, A. J. Percolation, Tie-Molecules, and the Microstructural Determinants of Charge Transport in Semicrystalline Conjugated Polymers. ACS Macro Lett. 2015, 4, 708-712.

(13) Sweetnam, S.; Vandewal, K.; Cho, E.; Risko, C.; Coropceanu, V.; Salleo, A.; Brédas, J. L.; McGehee, M. D. Characterizing the Polymer:Fullerene Intermolecular Interactions. Chem. Mater. 2016, 28, 1446-1452.

(14) Peumans, P.; Uchida, S.; Forrest, S. R. Efficient bulk heterojunction photovoltaic cells using small-molecular-weight organic thin films. Nature 2003, 425, 158-162.

(15) Robinson, D.; Besley, N. A. Modelling the spectroscopy and dynamics of plastocyanin. Phys. Chem. Chem. Phys. 2010, 12, 9667-9676.

(16) Abramavicius, D.; Jiang, J.; Bulheller, B. M.; Hirst, J. D.; Mukamel, S. Simulation Study of Chiral Two-Dimensional Ultraviolet Spectroscopy of the Protein Backbone. J. Am. Chem. Soc. 2010, 132, 7769-7775.

(17) Aghtar, M.; Kleinekathöfer, U.; Curutchet, C.; Mennucci, B. Impact of Electronic Fluctuations and Their Description on the Exciton Dynamics in the Light-Harvesting Complex PE545. J. Phys. Chem. B 2017, 121, 1330-1339.

(18) Ma, H.; Qin, T.; Troisi, A. Electronic excited states in amorphous MEH-PPV polymers from large-scale first principles calculations. J. Chem. Theory Comput. 2014, 10, 12721282.

(19) Wildman, J.; Repiščák, P.; Paterson, M. J.; Galbraith, I. General Force-Field Parametrization Scheme for Molecular Dynamics Simulations of Conjugated Materials in Solution. J. Chem. Theory Comput. 2016, 12, 3813-3824.

(20) Andreussi, O.; Prandi, I. G.; Campetella, M.; Prampolini, G.; Mennucci, B. Classical 
Force Fields Tailored for QM Applications: Is It Really a Feasible Strategy? J. Chem. Theory Comput. 2017, 13, 4636-4648.

(21) Claridge, K.; Troisi, A. Developing Consistent Molecular Dynamics Force Fields for Biological Chromophores via Force Matching. J. Phys. Chem. B 2019, 123, 428-438.

(22) Vanommeslaeghe, K.; Hatcher, E.; Acharya, C.; Kundu, S.; Zhong, S.; Shim, J.; Darian, E.; Guvench, O.; Lopes, P.; Vorobyov, I.; Mackerell Jr., A. D. CHARMM General Force Field. J. Comput. Chem. 2010, 31, 671-690.

(23) Wang, J.; Wolf, R. M.; Caldwell, J. W.; Kollman, P. A.; Case, D. A. Development and testing of a general Amber force field. J. Comput. Chem. 2004, 25, 1157-1174.

(24) Dodda, L. S.; De Vaca, I. C.; Tirado-Rives, J.; Jorgensen, W. L. LigParGen web server: An automatic OPLS-AA parameter generator for organic ligands. Nucleic Acids Res. 2017, 45, W331-W336.

(25) Huang, L.; Roux, B. Automated force field parameterization for nonpolarizable and polarizable atomic models based on ab initio target data. J. Chem. Theory Comput. 2013, 9, 3543-3556.

(26) Kreisbeck, C.; Kramer, T. Long-lived electronic coherence in dissipative exciton dynamics of light-harvesting complexes. J. Phys. Chem. Lett. 2012, 3, 2828-2833.

(27) Kim, C. W.; Park, J. W.; Rhee, Y. M. Effect of Chromophore Potential Model on the Description of Exciton-Phonon Interactions. J. Phys. Chem. Lett. 2015, 6, 2875-2880.

(28) Chandrasekaran, S.; Aghtar, M.; Valleau, S.; Aspuru-Guzik, A.; Kleinekathöfer, U. Influence of Force Fields and Quantum Chemistry Approach on Spectral Densities of BChl a in Solution and in FMO Proteins. J. Phys. Chem. B 2015, 119, 9995-10004.

(29) Prandi, I. G.; Viani, L.; Andreussi, O.; Mennucci, B. Combining classical molecular 
dynamics and quantum mechanical methods for the description of electronic excitations: The case of carotenoids. J. Comput. Chem. 2016, 37, 981-991.

(30) Ercolessi, F.; Adams, J. B. Interatomic Potentials from First-Principles Calculations: The Force-Matching Method. EPL 1994, 26, 583-588.

(31) Akin-Ojo, O.; Song, Y.; Wang, F. Developing ab initio quality force fields from condensed phase quantum-mechanics/molecular-mechanics calculations through the adaptive force matching method. J. Chem. Phys. 2008, 129, 064108.

(32) Wei, D.; Song, Y.; Wang, F. A simple molecular mechanics potential for $\mu$ m scale graphene simulations from the adaptive force matching method. J. Chem. Phys. 2011, $134,184704$.

(33) Wu, R.; Lu, Z.; Cao, Z.; Zhang, Y. A transferable nonbonded pairwise force field to model zinc interactions in metalloproteins. J. Chem. Theory Comput. 2011, 7, 433-443.

(34) Handley, C. M.; Deeth, R. J. A multi-objective approach to force field optimization: Structures and spin state energetics of d 6 Fe(II) complexes. J. Chem. Theory Comput. 2012, 8, 194-202.

(35) Wright, L. B.; Rodger, P. M.; Walsh, T. R. Aqueous citrate: A first-principles and force-field molecular dynamics study. RSC Adv. 2013, 3, 16399-16409.

(36) Sala, J.; Guàrdia, E.; Masia, M. Improving the force matching algorithm: Application to a simple point charge flexible model of water. Comput Phys Commun 2011, 182, $1954-1957$.

(37) Gabrieli, A.; Sant, M.; Demontis, P.; Suffritti, G. B. Fast and efficient optimization of Molecular Dynamics force fields for microporous materials: Bonded interactions via force matching. Microporous Mesoporous Mater. 2014, 197, 339-347. 
(38) Koziol, L.; Fried, L. E.; Goldman, N. Using Force Matching To Determine Reactive Force Fields for Water under Extreme Thermodynamic Conditions. J. Chem. Theory Comput. 2017, 13, 135-146.

(39) Lindsey, R. K.; Fried, L. E.; Goldman, N. Application of the ChIMES Force Field to Nonreactive Molecular Systems: Water at Ambient Conditions. J. Chem. Theory Comput. 2019, 15, 436-447.

(40) Li, J.; Wang, F. Water graphene contact surface investigated by pairwise potentials from force-matching PAW-PBE with dispersion correction. J. Chem. Phys. 2017, 146, 054702 .

(41) Lindsey, R. K.; Fried, L. E.; Goldman, N. ChIMES: A Force Matched Potential with Explicit Three-Body Interactions for Molten Carbon. J. Chem. Theory Comput. 2017, 13, 6222-6229.

(42) Do, H.; Troisi, A. Developing accurate molecular mechanics force fields for conjugated molecular systems. Phys. Chem. Chem. Phys. 2015, 17, 25123-25132.

(43) Chandran, D.; Lee, K.-S. Diketopyrrolopyrrole: A versatile building block for organic photovoltaic materials. Macromol Res 2013, 21, 272-283.

(44) Kanibolotsky, A. L.; Findlay, N. J.; Skabara, P. J. Polythiophene and oligothiophene systems modified by TTF electroactive units for organic electronics. Beilstein J. Org. Chem. 2015, 11, 1749-1766.

(45) Jorgensen, W. L.; Maxwell, D. S.; Tirado-Rives, J. Development and Testing of the OPLS All-Atom Force Field on Conformational Energetics and Properties of Organic Liquids. J. Am. Chem. Soc. 1996, 118, 11225-11236.

(46) Robertson, M. J.; Tirado-Rives, J.; Jorgensen, W. L. Improved Peptide and Protein 
Torsional Energetics with the OPLS-AA Force Field. J. Chem. Theory Comput. 2015, 11, 3499-3509.

(47) Robertson, M. J.; Qian, Y.; Robinson, M. C.; Tirado-Rives, J.; Jorgensen, W. L. Development and Testing of the OPLS-AA/M Force Field for RNA. J. Chem. Theory Comput. 2019, 15, 2734-2742.

(48) Phillips, J. C.; Braun, R.; Wang, W.; Gumbart, J.; Tajkhorshid, E.; Villa, E.; Chipot, C.; Skeel, R. D.; Kalé, L.; Schulten, K. Scalable molecular dynamics with NAMD. J. Comput. Chem. 2005, 26, 1781-1802.

(49) Schlegel, H. B.; Millam, J. M.; Iyengar, S. S.; Voth, G. A.; Daniels, A. D.; Scuseria, G. E.; Frisch, M. J. Ab initio molecular dynamics: Propagating the density matrix with Gaussian orbitals. J. Chem. Phys. 2001, 114, 9758-9763.

(50) Iyengar, S. S.; Schlegel, H. B.; Millam, J. M.; A. Voth, G.; Scuseria, G. E.; Frisch, M. J. Ab initio molecular dynamics: Propagating the density matrix with Gaussian orbitals. II. Generalizations based on mass-weighting, idempotency, energy conservation and choice of initial conditions. J. Chem. Phys. 2001, 115, 10291.

(51) Schlegel, H. B.; Iyengar, S. S.; Li, X.; Millam, J. M.; Voth, G. A.; Scuseria, G. E.; Frisch, M. J. Ab initio molecular dynamics: Propagating the density matrix with Gaussian orbitals. III. Comparison with Born-Oppenheimer dynamics. J. Chem. Phys. 2002, $117,8694-8704$.

(52) Frisch, M. J. et al. Gaussian 16, Revision C.01. Gaussian, Inc. Wallingford, CT, 2016.

(53) Lu, T.; Chen, F. Multiwfn: a multifunctional wavefunction analyzer. J. Comput. Chem. 2012, 33, 580-592.

(54) Peach, M. J.; Benfield, P.; Helgaker, T.; Tozer, D. J. Excitation energies in density 
functional theory: An evaluation and a diagnostic test. J. Chem. Phys. 2008, 128, 044118.

(55) Guido, C. A.; Cortona, P.; Mennucci, B.; Adamo, C. On the metric of charge transfer molecular excitations: a simple chemical descriptor. J. Chem. Theory Comput. 2013, 9, 3118-3126.

(56) Bronstein, H.; Collado-Fregoso, E.; Hadipour, A.; Soon, Y. W.; Huang, Z.; Dimitrov, S. D.; Ashraf, R. S.; Rand, B. P.; Watkins, S. E.; Tuladhar, P. S.; Meager, I.; Durrant, J. R.; McCulloch, I. Thieno[3,2- b ]thiophene-diketopyrrolopyrrole Containing Polymers for Inverted Solar Cells Devices with High Short Circuit Currents. Adv. Funct. Mater. 2013, 23, 5647-5654.

(57) Meager, I.; Ashraf, R. S.; Rossbauer, S.; Bronstein, H.; Donaghey, J. E.; Marshall, J.; Schroeder, B. C.; Heeney, M.; Anthopoulos, T. D.; McCulloch, I. Alkyl Chain Extension as a Route to Novel Thieno[3,2- b ]thiophene Flanked Diketopyrrolopyrrole Polymers for Use in Organic Solar Cells and Field Effect Transistors. Macromolecules 2013, 46, $5961-5967$.

(58) Shin, J.; Park, G. E.; Lee, D. H.; Um, H. A.; Lee, T. W.; Cho, M. J.; Choi, D. H. Bis(thienothiophenyl) Diketopyrrolopyrrole-Based Conjugated Polymers with Various Branched Alkyl Side Chains and Their Applications in Thin-Film Transistors and Polymer Solar Cells. ACS Appl. Mater. Interfaces 2015, 7, 3280-3288.

(59) Pop, F.; Lewis, W.; Amabilino, D. B. Solid state supramolecular structure of diketopyrrolopyrrole chromophores: correlating stacking geometry with visible light absorption. CrystEngComm 2016, 18, 8933-8943.

(60) Jorgensen, W. L.; Tirado-Rives, J. The OPLS [optimized potentials for liquid simulations] potential functions for proteins, energy minimizations for crystals of cyclic peptides and crambin. J. Am. Chem. Soc. 1988, 110, 1657-1666. 
(61) Bhatta, R. S.; Tsige, M. Chain length and torsional dependence of exciton binding energies in P3HT and PTB7 conjugated polymers: A first-principles study. Polymer 2014, 55, 2667-2672.

(62) Cornell, W. D.; Cieplak, P.; Bayly, C. I.; Kollman, P. A. Application of RESP charges to calculate conformational energies, hydrogen bond energies and free energies of solvation. J. Am. Chem. Soc. 1993, 115, 9620-9631.

(63) Siepmann, J. I.; Frenkel, D. Configurational bias Monte Carlo: a new sampling scheme for flexible chains. Mol Phys 1992, 75, 59-70.

(64) Hoover, W. G.; Ladd, A. J. C.; Moran, B. High-Strain-Rate Plastic Flow Studied via Nonequilibrium Molecular Dynamics. Phys. Rev. Lett. 1982, 48, 1818-1820.

(65) Herce, H. D.; Garcia, A. E.; Darden, T. The Electrostatic Surface Term: (I) Periodic Systems. J. Chem. Phys. 2007, 126, 124106.

(66) Kawanabe, Y.; Moulé, A. J.; Faller, R. Molecular Dynamics Study of the Local Structure of Photovoltaic Polymer PCDTBT. J. Chem. Eng. Data 2014, 59, 2982-2986.

(67) Le Bahers, T.; Adamo, C.; Ciofini, I. A qualitative index of spatial extent in chargetransfer excitations. J. Chem. Theory Comput. 2011, 7, 2498-2506.

(68) Peach, M. J.; Le Sueur, C. R.; Ruud, K.; Guillaume, M.; Tozer, D. J. TDDFT diagnostic testing and functional assessment for triazene chromophores. Phys. Chem. Chem. Phys. 2009, 11, 4465-4470.

(69) Köhler, A.; Hoffmann, S. T.; Bässler, H. An order-disorder transition in the conjugated polymer MEH-PPV. J. Am. Chem. Soc. 2012, 134, 11594-11601.

(70) Aragó, J.; Troisi, A. Dynamics of the excitonic coupling in organic crystals. Phys. Rev. Lett. 2015, 114, 026402. 
(71) Yamagata, H.; Norton, J.; Hontz, E.; Olivier, Y.; Beljonne, D.; Brédas, J.-L.; Silbey, R.; Spano, F. The nature of singlet excitons in oligoacene molecular crystals. J. Chem. Phys. 2011, 134, 204703.

(72) Aragó, J.; Troisi, A. Regimes of exciton transport in molecular crystals in the presence of dynamic disorder. Adv. Funct. Mater. 2016, 26, 2316-2325.

(73) Fornari, R. P.; Aragó, J.; Troisi, A. Exciton dynamics in phthalocyanine molecular crystals. J. Phys. Chem. C 2016, 120, 7987-7996. 
Graphical TOC Entry

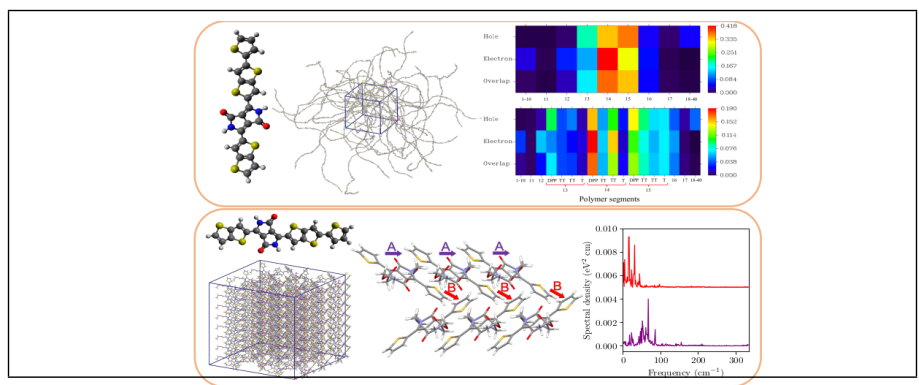

